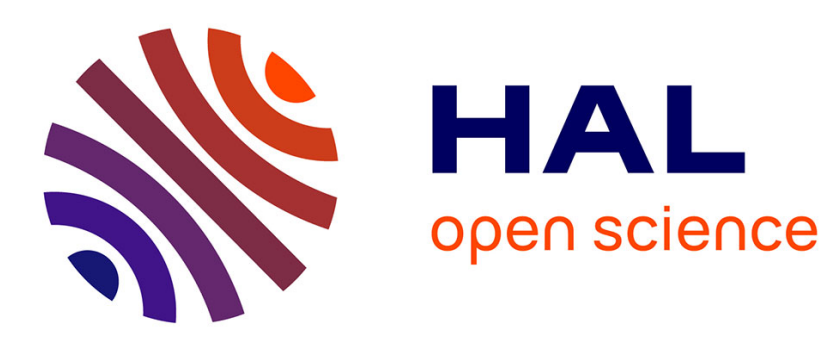

\title{
Planesiai, îles erratiques de l'Occident grec
}

Pierre Moret

\section{- To cite this version:}

Pierre Moret. Planesiai, îles erratiques de l'Occident grec. Revue des Etudes Grecques, 1997, 110 (1), p. 25-56. hal-00723948

\section{HAL Id: hal-00723948 \\ https://hal.science/hal-00723948}

Submitted on 23 Aug 2012

HAL is a multi-disciplinary open access archive for the deposit and dissemination of scientific research documents, whether they are published or not. The documents may come from teaching and research institutions in France or abroad, or from public or private research centers.
L'archive ouverte pluridisciplinaire HAL, est destinée au dépôt et à la diffusion de documents scientifiques de niveau recherche, publiés ou non, émanant des établissements d'enseignement et de recherche français ou étrangers, des laboratoires publics ou privés. 


\section{PLANESIAI, ÎLES ERRATIQUES DE L'OCCIDENT GREC}

RÉsumÉ. - Trois îles de la Méditerranée occidentale, Pianosa en mer Tyrrhénienne, Saint-Honorat en Ligurie et Tabarca en Ibérie, portaient dans l'Antiquité le même nom grec, Planesia ou Planasia, qui signifiait «l'île errante». Sur le littoral maurétanien, l'Insula Erroris mentionnée par une source latine se rattache sans doute à la même famille toponymique. L'arrière-fond mythique de la notion d'île errante ou mouvante est ensuite étudié. L'intervention d'un dieu qui immobilise et enracine l'île vagabonde est attestée à Délos - dont le premier nom, Asteria, a les mêmes connotations astrales que Planesia -, mais aussi à Tyr, sous les espèces d'un Héraclès-Melqart fortement hellénisé. Ces mythes de fondation autorisent une nouvelle lecture de la légende qui montre saint Honorat chassant les serpents et faisant sourdre l'eau douce sur l'île primitivement errante de Lérins.

Abstract. - Three islands of the Western Mediterranean, Pianosa in the Tyrrhenian sea, Saint-Honorat in Liguria and Tabarca in Iberia, were called in hellenistic times by the same greek name, Planesia or Planasia, which means "the wandering island». Insula Erroris, on the Mauretanian coast, probably belongs to the same toponymic family. The mythical background of the floating or wandering islands is then explored. The figure of a god who brings the roaming island to a standstill plays a crucial part in Delos whose former name, Asteria, has the same astral connotations as Planesia - and in the hellenistic Tyre. These foundation myths support a comparison with the christian legend that shows saint Honorat driving the snakes away and causing a spring of sweet water to gush forth in the "wandering island» of Lérins.

Trois îles de la Méditerranée occidentale, réparties entre l'Ibérie, la Ligurie et l'Étrurie, ont été appelées Planesia ou Planasia par les Anciens. Cette rencontre de trois noms identiques m'intriguait depuis longtemps, et plus encore le fait que ces îles sont toutes situées à proximité d'une fondation phocéenne ou marseillaise. L'île ligure est en effet voisine d'Antibes-Antipolis, l'île toscane guère éloignée d'Aléria-Alalia, et l'île ibère en vue d'Alônis, "ville et île de Marseille» d'après Artémidore'. Un nom d'apparence hellénique, répété comme en écho sur les bords de la même

I Nous reviendrons plus loin sur les difficultés que soulève cette trop brève définition. 
région maritime, et qui semblait jalonner d'île en île l'aventure des Grecs dans l'Extrême-Occident : c'était assez pour soumettre Planesia à une enquête étymologique de routine.

Telle était du moins mon intention première; car cette enquête devait m'entraîner presque malgré moi à la poursuite des "îles errantes" que sont, nous le verrons, les îles Planésies, dans un périple littéraire qui m'ouvrait à chaque escale des vues insoupçonnées, de Délos aux roches Planctes et de Tyr à Gadès, et plaçait tour à tour sur ma route les silhouettes d'une héroïne de Plaute, d'Héraclès et de saint Honorat. Mais ne quittons pas trop vite nos trois ports d'attache; avant d'aborder les questions passablement complexes de l'étymologie, du sens et de l'arrière-fond mythique du mot Planesia, il m'a paru nécessaire de faire un point rapide sur la géographie et la première histoire des îles qui se sont partagé ce nom.

\section{En Ibérie : Tabarca}

La petite île de Tabarca - officiellement, Isla de Nueva Tabarca - fait face au cap de Santa Pola (province d'Alicante), à quatre kilomètres de la côte. Ses 23 hectares de terre aride, impropre aux cultures, s'étirent sur plus d'un kilomètre et demi d'est en ouest $^{2}$. Son nom actuel garde le souvenir des tribulations de six cents corailleurs génois, razziés par les Barbaresques sur l'île tunisienne de Tabarca, où ils exerçaient leur industrie; le roi d'Espagne Charles III racheta leur liberté en 1768 et les installa sur cette île alors déserte, qui semblait favorable à la pêche du corail, et qui reçut le nom de «Nouvelle Tabarca». L'appellation Isla de Santa Pola, du nom du port le plus proche sur le continent, avait été jusque là la plus courante ${ }^{3}$. Mais l'île portait un autre nom, celui d'Isla Plana, "l'île plate», qui se conserva quelque temps et qui, quoique aujourd'hui tombé en désuétude, se lit encore sur des cartes géographiques récentes. L'étymologie de ce troisième nom, parfaitement en accord avec le profil aplani de l'île, semble transparente. Mais dès le XVIII $^{\mathrm{e}}$ siècle, la sagacité des érudits locaux avait, de proche en proche, en remontant du catalan médiéval à

\footnotetext{
${ }^{2}$ Sur l'île de Tabarca, d'un point de vue général : A. Ramos Folques, La Isla de Tabarca, Alicante, 1970 ; J.F. Mullet Pedrós et H. Lillo García, Isla de Tabarca, Alicante, 1989.

${ }^{3}$ C'est le nom officiel de l'île entre le $\mathrm{XIV}^{\mathrm{e}}$ et la première moitié du XvIII ${ }^{\mathrm{e}}$ siècle. Cf. J.A. Mayans i Siscar, Ilici, hoi la villa de Elche, ilustrada con varios discursos, Valencia, 1771 , p. 161,205 et 208.
} 
l'arabe puis au grec, suivi le fil d'une étymologie plus ancienne et plus complexe, qui nous mène jusqu'à Strabon.

Strabon mentionne en effet, dans sa brève description du littoral

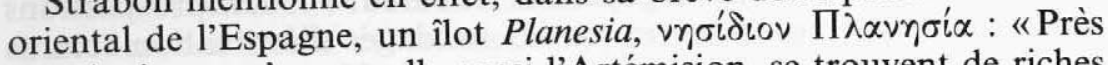
de Dianium, qu'on appelle aussi l'Artémision, se trouvent de riches mines de fer et les îlots de Planesia et de Plumbaria, puis, à l'intérieur, une lagune de quatre cents stades de pourtour $»{ }^{4}$. Le cadre géographique vaguement indiqué par Strabon autorise à situer l'îlot Planesia quelque part entre Denia (l'antique Dianium) et le Mar Menor. Dans cette portion de côte, il n'existe que quatre îles ou îlots dignes de mention : du nord au sud, l'île de Portichol, l'îlot de Benidorm, l'île de Tabarca et l'Isla Grosa.

Nous serions bien en peine de distinguer parmi ces quatre îles celle qui porta le nom de Planesia, si nous ne savions par le récit du voyageur et géographe arabe Al-Idrîsî que l'île de Tabarca s'appelait, au XII ${ }^{\mathrm{e}}$ siècle, Ablanasa ${ }^{5}$. Compte tenu des particularités de la phonétique arabe $^{6}$, ce nom est le calque transparent du toponyme grec, qui dut rester en usage, sous une forme latinisée (Planesia ou Planasia), jusqu'à l'Antiquité tardive. La graphie initiale Plan- refait d'ailleurs surface après la Reconquête : d'abord sous la forme Planesa ${ }^{7}$, encore très proche de l'original, puis, comme nous l'avons vu, sous la forme abrégée Plana qui survivra jusqu'à l'époque contemporaine. Cette dernière évolution est due à une confusion étymologique : dans le dialecte valencien, dérivé du catalan, qui s'impose avec la Reconquête, le toponyme hérité des Arabes est tout naturellement compris comme une référence à la planéité de l'île ${ }^{8}$. Quant au sens véritable du mot grec, la question

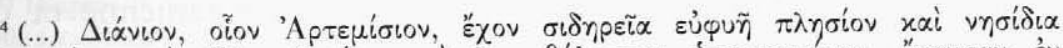

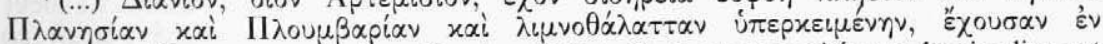

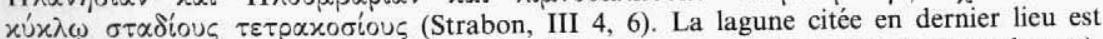
le Mar Menor. Strabon mentionne ensuite l'île de Scombroaria (auj. Isla de Escombreras), proche de Carthagène.

${ }^{5}$ «Près de cette ville [scil. Alicante], à l'ouest, il y a une île qui s'appelle Ablanasa, située à un mille de la côte" (Al-Idrîsî, Opus Geographicum, Naples-Rome, 1975, texte arabe p. 558). Cf. C.E. Dubler, "Al-Andalus en la geografía de al-Idrîsî", Studi Maghrebini, XX, 1988, p. 128. Je remercie vivement Patrice Cressier et Mohammed Meouak pour la traduction du passage décrivant Planesia et pour leurs indications concernant la graphie exacte du mot arabe.

${ }^{6}$ Le son $\mathrm{p}$, inconnu en arabe, est suppléé par un b; quant à la voyelle initiale, il s'agit d'un alif prosthétique. On sait en effet que l'arabe n'admet pas en début de mot une syllabe commençant par deux consonnes consécutives.

7 « La Isla de Santa Pola (...) hoi dia se llama por otro nombre la Isla Planesa, por la llanura que tiene» (Gaspar Escolano, qui écrit en 1610, cité par Mayans, op. cit., p. 203).

${ }^{8}$ Les grandes lignes de cette tortueuse histoire, du grec au catalan, n'avaient pas échappé à Mayans : «Los Marselleses, colonos de los Griegos Foceenses, pusieron a esta Isla el nombre de Planasia (...). Esto corrompieron los Arabes en el de Palnatsa. Los Lemosines [i. e. les Catalans], viendo este vocablo, la nombraron Planesa, en consi- 
est plus complexe et demande des développements qui n'auraient pas leur place ici; nous y viendrons tout à l'heure.

Les recherches archéologiques à Tabarca - des prospections dans l'immédiat après-guerre, et des sondages d'évaluation récents - n'ont pas livré de vestiges antérieurs au Haut-Empire ${ }^{9}$; encore ces vestiges ne prouvent-ils pas une occupation constante, mais peut-être, tout au plus, l'existence de campements de pêcheurs. Rappelons qu'avant l'installation des six cents Génois, qui exigea d'importants travaux d'infrastructure de la part de l'Etat espagnol, l'île était inhabitée ${ }^{10}$.

\section{En Ligurie : Lérins}

Les îles de Lérins, qui prolongent la pointe de la Croisette entre le golfe de la Napoule et le golfe Juan, ont été rendues fameuses par leur monastère qui fut l'un des premiers et l'un des plus brillants de la Gaule chrétienne; mais elles étaient déjà bien connues des Grecs. Elles sont deux, de taille et de physionomie contrastées. Au nord, l'île Sainte-Marguerite est la plus grande et la plus élevée. Elle possède un bon port en eau calme. Au sud, la petite île SaintHonorat (70 hectares) est basse et d'accès difficile, avec des côtes entièrement rocheuses. Ces conditions peu favorables sont balancées par la vigueur de sa végétation ${ }^{11}$ et par l'existence d'une source d'eau douce qui rendit possible, aux temps de splendeur de l'abbaye, l'installation d'une nombreuse communauté monastique $^{12}$. Seule l'île Sainte-Marguerite a fait l'objet de recherches archéologiques approfondies. Toutes les constructions mises au jour sont romaines; une occupation antérieure est cependant attestée par de nombreux tessons de céramique campanienne et des

deración del sitio natural, que es llano.» (op. cit., p. 204). Nous verrons plus loin ce qu'il faut penser du rôle prêté aux Marseillais par l'ecclésiastique illicitain.

9 J. Belda, "Mapa arqueológico de la Isla de Tabarca», Papeles de Oro, 9, Alicante, 1970 ; P. Rosser Limiñana, "Memoria de las actuaciones realizadas en el ejercicio 19921993 y principios de 1994 ", LQNT - Patrimonio Cultural de la Ciudad de Alicante, 2 ,
1994 , p. 11-34.

${ }^{10}$ Mayans croit même qu'elle n'avait jamais été habitée (op. cit., p. 201). Opinion partagée par un chroniqueur du XVII ${ }^{\mathrm{e}}$ siècle: "No ha sido nunca habitada, aunque me dicen que hay vestigios que debieron ser de alguna atalaya o abrigo de pescadores" (V. Bendicho, Crónica de la muy ilustre, noble y leal ciudad de Alicante, ms. de 1640 publié par F. Figueras Pacheco, Alicante, 1960, p. 27).

11 En 1635, les Espagnols s'emparent de l'île et coupent les bois de pins qui l'ombrageaient: "cette forêt faisait donner à Lérins par les mariniers le nom d'Aigrette de la Mer" (Morery, Dictionnaire historique, Paris, 1704, s.v. Lérins).

${ }^{12} 500$ moines vivaient à Saint-Honorat lorsque l'île fut prise par les Sarrasins, en 730 (R.P. Hélyot, Dictionnaire des ordres religieux, II, Paris, 1847, p. 770). 
poteries de l'âge du fer indigène. La céramique attique est absente dans les dépôts fouillés ${ }^{13}$.

Pline l'Ancien et l'Itinéraire maritime d'Antonin ${ }^{14}$ donnent à ces deux îles des noms étroitement apparentés, Lero et Lerina, qui déclinent la même racine au masculin et au féminin ${ }^{15}$. Strabon, qui reprend probablement la substance d'une description de Posidonios, se distingue en donnant le nom de Planasie à l'une des deux îles ${ }^{16}$ : «Après les Stœchades, on trouve les îles de Planasia et de Lérôn, qui sont habitées ${ }^{17}$. Il y a aussi à Lérôn un sanctuaire du héros Lérôn. Cette île fait face à Antipolis ». Un ex-voto inscrit, récemment découvert à Sainte-Marguerite ${ }^{18}$, confirme à la fois la réalité du culte évoqué par Strabon et l'authenticité des noms transmis par Pline.

Nous avons donc trois noms pour deux îles. Le fait que Planasia soit associé à Lerôn dans la description de Strabon implique nécessairement que Planasia et Lerina désignent la même île, comme on pourra s'en convaincre à la lecture du tableau suivant :

$\begin{array}{lll}\text { Strabon } & \text { Lérôn } & \text { Planasia } \\ \text { Ex-voto } & \text { Lérôn } & \text { Lériné } \\ \text { Pline } & \text { Lero }(n) & \text { Lerina } \\ \text { Itin. Marit. } & \text { Lero }(n) & \text { Lerin }<a>\text { (mss. Lerino) } \\ \text { Ptolémée } & \text { Lérôné } & \text { - }\end{array}$

${ }^{13} \mathrm{~J}$. Formigé, "La station antique de Lero à l'île Sainte-Marguerite (Alpes-Maritimes)", Gallia, 5, 1947, p. 146-155 ; J. Coupry et G. Vindry, "Lérôn et Lériné aux îles de Lérins. Un couvercle en ivoire, à dédicace grecque, découvert à l'île Sainte-Marguerite (Cannes)", RAN, 15, 1982, p. 353-358.

${ }^{14}$ Pline, III $79:<L>$ ero et Lerina adversum Antipolim, in qua Berconi oppidi memoria. Itinéraire Maritime, p. 504 (ed. Wesseling) : Ab Antipoli Lero et Lerino insulae, mpm XI.

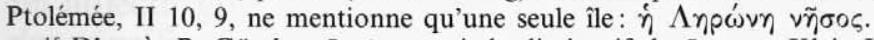

15 D'après P. Gössler, Lerina serait le diminutif de Lero : "Klein-Lero" ( $R E, \mathrm{XX}, 2$, 1950 , s.v. Planasia). En fait, le morphème -ina est ici simplement la marque du féminin

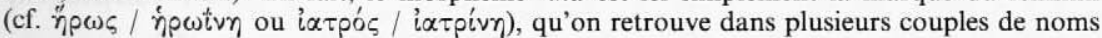
propres grecs (cf. P. Chantraine, La formation des noms en grec ancien, Paris, 1933, p. 205, et C.D. Buck \& W. Petersen, A Reverse Index of Greek Nouns and Adjectives, Chicago, 1948 , p. 288).

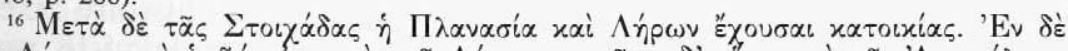

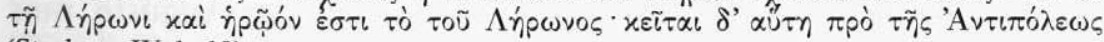
(Strabon, IV 1, 10).

${ }^{17}$ J'adopte ici la traduction de Lasserre, qui respecte l'imprécision (regrettable!) du

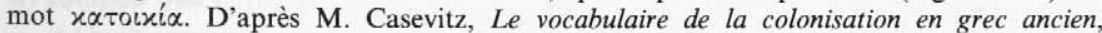
Paris, 1985, p. 164, ce mot désigne sauf contextes particuliers «une localité de moyenne ou petite importance et en général tout établissement d'une population peu organisée». Pour les Stœchades, plus grandes et plus fertiles, Strabon utilise une formule plus précise, $\gamma \varepsilon \omega p \gamma \sigma \tilde{\sigma} \sigma \delta^{\prime} \alpha \cup ่ \dot{\alpha} \zeta \quad M \propto \sigma \sigma \alpha \lambda \iota \tilde{\omega} \tau \alpha \iota$, qui met l'accent sur la mise en valeur agricole des iles et sur leur appartenance au domaine marseillais.

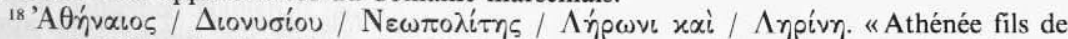
Denys, de Néôpolis, à Lérôn et à Lériné " (Coupry et Vindry, op. cit.). Seule différence par rapport à l'allusion de Strabon: c'est au couple éponyme, protecteur des deux îles, qu'un culte est rendu, non au seul Lérôn. L'objet provient de niveaux hellénistiques (Iv ${ }^{\mathrm{e}}$ II $^{\mathrm{e}}$ s. av. J.-C.). 
La tradition littéraire chrétienne nous permet d'identifier sans risque d'erreur cette île que les Anciens connurent sous les deux noms de Planasia et de Lerina. En 410, le monastère de Lérins est fondé par saint Honorat sur une île que les auteurs du $\mathrm{v}^{\mathrm{e}}$ siècle nomment Lerina, Lerinus, Lirinus ou Lirina ${ }^{19}$. Le maintien du thème en -i- dans toutes ces variantes, mêmes dans celles qui se déclinent au masculin (par opposition au thème en -o- de la série Lero / Lérôn), prouve que la Lériné hellénistique, la Lerina romaine et la moderne Saint-Honorat, siège du monastère, sont une seule et même île.

Dans leurs grandes lignes, les faits sont donc simples : Lero est devenu Sainte-Marguerite, et Lerina Saint-Honorat (au prix, d'ailleurs, d'une curieuse inversion de genre). Mais qu'en est-il de Planasia? Si Posidonios est bien la source du passage de Strabon qui nous intéresse, il faut admettre que Planasia était le seul nom ou le nom le plus courant de l'île Saint-Honorat à l'époque où Posidonios séjourna à Marseille et dans sa région, soit entre 101 et 91 av. J.-C. ${ }^{20}$. Dans tous les cas, qu'il remonte ou non à Posidonios, le témoignage transmis par Strabon a toutes les chances d'être postérieur à la mention d'une divinité Lériné dans l'inscription hellénistique de Sainte-Marguerite. Théonyme, Lériné est peut-être aussi, dès cette époque, le second nom de l'île, en concurrence, donc, avec Planasia, avant de se substituer complètement à lui. Mais la chronologie de cette supplantation nous échappe. Seul fait tangible : le consensus des sources romaines, qui invite à penser que Planasia était tombé dans l'oubli, ou était passé au second plan, dès le premier siècle de notre ère.

\section{En Étrurie : Pianosa}

L'île tyrrhénienne de Planasia, aujourd'hui Pianosa (Planose ou Planouse dans les portulans français du XVIII ${ }^{\mathrm{e}}$ siècle), est la seule de nos îles homonymes qui ait laissé une trace dans l'histoire : c'est là que le malheureux Agrippa Postumus fut exilé par Auguste, puis exécuté sur ordre de Tibère en 14 après J.-C. ${ }^{21}$. C'est aussi celle dont l'identification est la moins douteuse. Son nom s'est conservé

\footnotetext{
19 Lirina insula en 430 dans saint Hilaire, Sermo de vita sancti Honorati (Patrologie, L, 1859, col. 1249-1272) ; Lerinus vers 420 dans Paulin de Nole, Epist. 51, 2 ; Lirinus après 460 dans Sidoine Apollinaire, Carm. XVI, 104. p. 67

${ }^{20}$ Sur la date de ce séjour, voir M. Laffranque, Poseidonios d'Apamée, Paris, 1964,

${ }_{21}$ Tacite, Annales, I 3, 4; Suétone, Auguste, 65; Dion Cassius, LV 32, 2.
} 
presque intact jusqu'à nos jours; elle est en outre citée et située par de nombreuses sources d'époque romaine ${ }^{22}$.

Nettement plus grande que les deux îles précédentes $\left(10,25 \mathrm{~km}^{2}\right)$, elle fait partie de l'archipel toscan, à $14 \mathrm{~km}$ au sud de l'île d'Elbe et à $45 \mathrm{~km}$ environ à l'est de la Corse. C'est une table calcaire grossièrement triangulaire, d'aspect bas et uniforme, culminant à $26 \mathrm{~m}$ d'altitude; ses côtes sont découpées, souvent rocheuses ${ }^{23}$. Au cours de son histoire, Pianosa a traversé des phases successives d'abandon et de colonisation. Son sol, modérément fertile, se prête surtout à la culture de la vigne et de l'olivier. Aucun vestige grec n'y a été signalé, alors que des traces d'occupation à l'époque romaine impériale ont été relevées en plusieurs points de l'île ${ }^{24}$.

\section{Parmi les îles Fortunées}

Pline nous a conservé la substance de deux descriptions des îles Fortunées - les modernes Canaries - : celle de Sebosus Statius, géographe romain antérieur à Néron, et celle de Juba II de Maurétanie. La première mentionne une Planasia ${ }^{25}$. On a pensé que ce nom, qui n'apparaît pas dans la liste de Juba, pouvait être le fruit d'une confusion ou d'une erreur de transcription du nom Canaria, attesté par Juba et qui s'est conservé jusqu'à nos jours ${ }^{26}$. Hypothèse toute gratuite, qui ne se prévaut que d'une vague consonance. Arrêtons-nous plutôt sur la glose de Pline : l'île s'appelle Planasia a specie, "d'après son aspect». Cela signifie que ce nom d'île était compris par Pline comme un adjectif descriptif, motivé par la forme ou le profil de l'île. Or, nous le verrons, Planasia est un adjectif grec lié au champ sémantique de l'errance, dont aucune des significations possibles ne convient à l'évocation d'un aspect physique. Pour que la glose prenne son sens, je ne vois d'autre

${ }^{22}$ Varron, Res rusticae, III 6, 2; Strabon, II 5, 19; Pline, III 81; Ptolémée, III 1, 79; Solin, III 2; Itinéraire d'Antonin, 514, 1 ; Anonyme de Ravenne, V 25; et les historiens cités à la note précédente.

${ }^{23}$ S. Sommier, "L'isola di Pianosa nel Mar Tirreno", Riv. Geogr. Italiana, XVI, 1909, p. $441-464,528-540$ et $585-606$.

${ }^{24}$ On se reportera sur ce sujet à la mise au point récente de L. Corsi dans G. Nenci et G. Vallet (dir.), Bibliografia topografica della colonizzazione greca in Italia e nelle isole Tirreniche, XIII, Pise et Rome, 1994, p. 535-546, avec toute la bibliographie antérieure.

${ }^{25}$ Hist. nat., VI 202 : ab iis CCL Fortunatas contra laevam Mauretaniae in VIII horam solis ; vocari Invallem a convexitate et Planasiam a specie.

${ }^{26}$ «Denselben Namen [Canaria] vermutet Partsch in des Sebosus Planasia (a specie), durch eine Irrtum des Plinius entstellt" (C. Th. Fischer, RE, VII (1), 1910, col. 43). V. Manfredi conclut plus prudemment à l'impossibilité d'identifier Planasia, et n'écarte pas l'éventualité d'une transcription inexacte (Le Isole Fortunate. Topografia di un mito, Rome, 1993, p. 97). 
solution que de corriger la leçon des manuscrits, en restituant le latin Planaria (l'île "plane») à la place du grec Planasia ${ }^{27}$.

Cette correction aurait en outre l'avantage d'unifier la toponymie des îles Fortunées. Les noms des quatre autres îles sont en effet tous latins dans Sebosus : Iunonia, Pluvialia, Capraria, Invallis. On sait que la connaissance précise des Canaries, à Rome et en Grèce, ne date que du $\mathrm{I}^{\mathrm{er}}$ siècle avant J.-C.; d'après Plutarque (Sert., 8), Sertorius aurait été le premier Romain instruit de leur existence. Il serait donc étonnant qu'un élément grec entrât dans la toponymie de ces îles atlantiques que les Phéniciens et les Puniques surent garder quasi secrètes jusqu'à la chute de Carthage ${ }^{28}$.

Le fait que le nom présente une suffixation grecque dans les manuscrits de Pline s'explique sans doute par l'attraction de la Planasia tyrrhénienne, devenue célèbre au premier siècle de notre ère comme lieu d'exil d'Agrippa Postumus. Dans cette hypothèse, la confusion serait plus probablement imputable à un copiste qu'à Pline lui-même. À moins d'admettre que c'est Pline lui-même qui a confondu le grec Planasia et le latin Planaria, par méconnaissance du sens étymologique de l'adjectif grec. Nous reviendrons plus loin sur cette éventualité.

Compte tenu de ces incertitudes et du contexte historique tardif des traditions géographiques concernant les îles Canaries, il m'a paru préférable de laisser cette Planasie atlantique à l'écart des réflexions qui vont suivre.

\section{Quelques points communs}

Pour qu'on leur ait donné le même nom, il faut bien supposer un point commun à ces trois îles. Mais la question n'est pas si simple : à première vue, ce sont les différences qui prédominent. SaintHonorat fait couple avec Sainte-Marguerite, alors que les deux autres îles sont isolées; Pianosa est plusieurs fois plus grande que Tabarca; Saint-Honorat est couverte d'arbres, Tabarca quasi désertique. Les affinités existent néanmoins, mais on ne peut les discerner qu'à condition de prendre le point de vue du navigateur, en replaçant ces îles dans leur contexte maritime.

${ }^{27}$ Ce serait revenir au texte des premiers éditeurs de Pline : leur choix n'était peut-être pas si mauvais.

${ }^{28}$ C'est seulement parce que Juba écrivait en grec qu'on trouve dans sa liste un Ombrion, qui est l'exacte traduction de Pluvialia. 


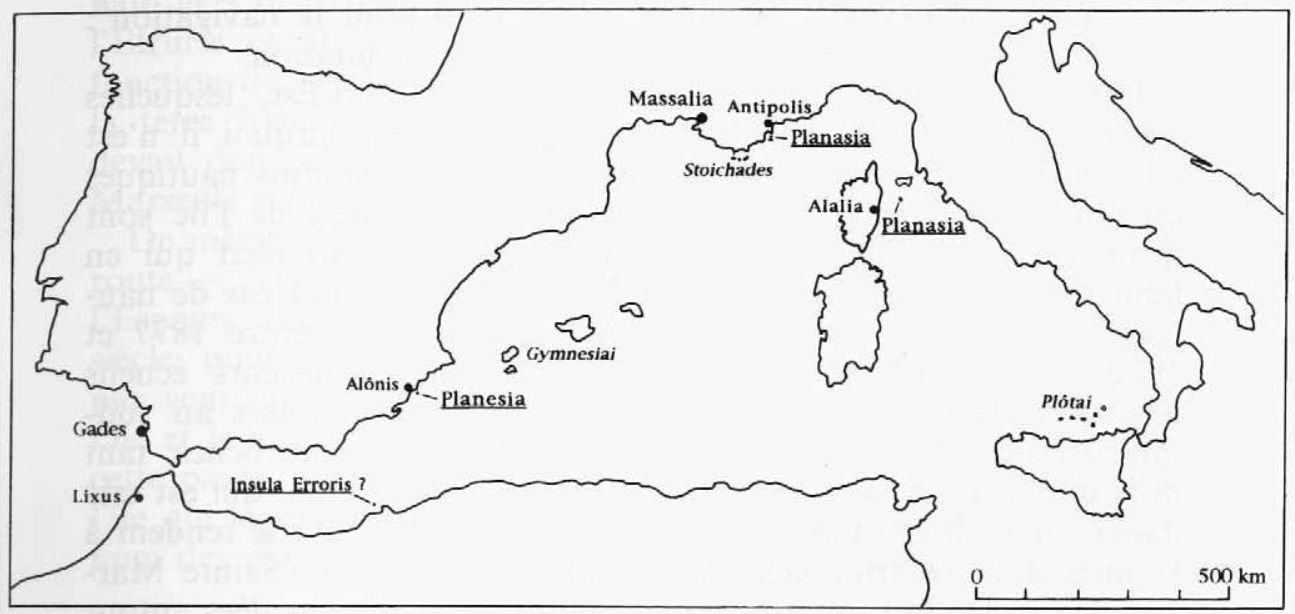

\section{Des îles peu fréquentées, aux abords dangereux}

Nous l'avons vu, Tabarca était inhabitée avant l'époque romaine; il est certes possible que l'île ait été fréquentée, mais ces visites ou ces brefs séjours n'ont pas laissé de restes assez consistants pour retenir l'attention des archéologues. À SaintHonorat, les données archéologiques sont insuffisantes; on devra se contenter des sources littéraires qui mentionnent, au $\mathrm{I}^{\mathrm{er}}$ siècle de notre ère, les ruines (littéralement : le souvenir) d'un oppidum Berconum ou Vergonum ${ }^{29}$, et aux environs de 100 av. J.-C., un simple habitat ${ }^{30}$. Dans tous les cas, la superficie exiguë de l'île ne pouvait s'accommoder que d'un peuplement réduit. Même discrétion à Pianosa, qui ne sort de l'anonymat qu'en devenant un lieu de déportation à l'époque romaine, au point qu'on est tenté de l'inclure dans le groupe d'îles inhabitées avoisinant l'île d'Elbe auquel fait allusion, vers le milieu du $\mathrm{IV}^{\mathrm{e}}$ siècle, le Pseudo-Scylax (Périple, 6).

Petites, peu fertiles - Tabarca est même absolument aride — et dépourvues de mouillages sûrs, ces trois îles n'étaient guère attirantes. Pour les marins grecs, elles ne furent sans doute jamais le but du voyage; ce n'étaient que des amers, des points de repère sur des routes qui, tout en longeant leurs côtes, les laissaient à l'écart.

${ }^{29}$ Pline, Hist. nat., III 79 (voir supra n. 14). Les mss. donnent berconi, bergoni, vergoni; aucune de ces leçons ne s'impose objectivement. La variante Vergoani, retenue dans plusieurs travaux récents d'archéologie provençale, est un lapsus des premiers éditeurs.

${ }^{30}$ Strabon, IV 1, 10, d'après Posidonios (voir supra n. 16). Cette allusion à des $x \alpha \tau o \iota-$ $x i \alpha \iota$ concerne les deux îles de Lérins prises ensemble. 
Mais elles constituaient aussi un sérieux péril pour la navigation : c'est à ce titre surtout qu'elles gagnèrent leur réputation.

L'île de Tabarca «a plusieurs roches au Sud-Est, lesquelles s'avancent bien une demi-lieue en mer : c'est pourquoi il n'est pas bon de la ranger de ce côté-là $»^{31}$. Les instructions nautiques espagnoles signalent que les extrémités est et ouest de l'île sont prolongées par des écueils, des hauts fonds et un récif qui en rendent les abords très dangereux ${ }^{32}$; on rapporte huit cas de naufrages ou d'échouages sur les écueils de Tabarca, entre 1877 et $1922^{33}$. L'île Saint-Honorat a, du côté sud, "plusieurs écueils dessus et dessous l'eau; et à environ trois cents toises au sudquart-sud-est de l'abbaye, l'on voit un petit banc de roches, tant hors de l'eau que sous l'eau, qu'on appelle les Moines, qui est très dangereux ». Il est donc recommandé aux bateaux qui se rendent à Cannes de "s'écarter des isles Saint Honoré (sic) et Sainte Marguerite ${ }^{34}$. De fait, quatre épaves antiques ont été signalées autour des îles de Lérins, dont une près du rocher des Moines ${ }^{35}$. Quant à Pianosa, elle «est à la rase de la mer, c'est pourquoi elle est fort dangereuse de nuit, car on serait dessus sans la voir ${ }^{36}$; sur de nombreux points de ses côtes des récifs, des bancs rocheux et des écueils couverts d'eau s'avancent jusqu'à un demi-mille au large ${ }^{37}$.

\section{Des îles situées sur des routes maritimes majeures...}

Sur le littoral provençal, les courants du circuit ligure, qui portent d'est en ouest, favorisent la navigation côtière. Les îles de Lérins étaient donc nécessairement rangées par la grande majorité des navires qui se rendaient d'Italie à Marseille, comme ce bateau marchand du ${ }^{\text {er }}$ siècle av. J.-C., coulé à deux milles des îles dans le golfe Juan, dont la cargaison montre qu'il venait d'un port de la côte tyrrhénienne ${ }^{38}$.

Pianosa se trouve dans une position comparable. La route maritime qui, venant de l'ouest (de Marseille ou des Baléares), contour-

${ }^{31}$ Le petit flambeau de la mer, Cabotage, ou Le véritable guide des pilotes côtiers, Paris, 1719 , p. 283.

${ }_{32}$ Derrotero del Mediterráneo, Madrid, 1920, p. 284.

${ }^{33}$ A. Ramos Folques, La Isla de Tabarca, Alicante, 1970, p. 70-71.

${ }^{34} \mathrm{H}$. Michelot, Portulan de la mer Méditerranée, ou Guide des pilotes côtiers, Marseille, 1824 , p. 186.

${ }^{35}$ A.J. Parker, Ancient Shipwrecks of the Mediterranean \& the Roman Provinces (B.A.R., Int. Ser., 580), Oxford, 1992, nº 97, 1004, 1010 et 1174.

${ }^{36}$ Le petit flambeau de la mer, op. cit., p. 319.

${ }^{37}$ Michelot, op. cit., p. 233.

${ }^{38} \mathrm{C}$. Baudoin et al., Une cargaison de bronzes hellénistiques. L'épave Fourmigue $C$ à Golfe-Juan (Archaeonautica, 12), Paris, 1994. 
nait la Corse par le nord, pour redescendre vers les côtes de l'Étrurie et du Latium ${ }^{39}$, pouvait emprunter deux passages, en fonction du régime saisonnier des vents : soit entre l'île d'Elbe et la terre ferme, soit entre l'île d'Elbe et Pianosa. Cette dernière devait donc être familière à tous les marins qui cabotaient entre Marseille et les ports de la mer Tyrrhénienne.

De même, Tabarca constituait un point de passage obligé sur la route - certes moins fréquentée - qui longeait la côte sud-est de l'Espagne. Les instructions nautiques recommandaient il y a un siècle, pour les navires à petit tirant d'eau qui venaient du nord par vent dominant d'est, d'emprunter le canal de Tabarca, entre l'île et la côte ${ }^{40}$. Le cabotage antique connaissait sans doute aussi cette passe, qui n'était pas sans dangers; il était donc naturel que l'île qui la signalait et qui en constituait l'une des limites reçût un nom des marins grecs.

\section{... et à proximité d'établissements phocéens}

Les trois îles Planésies sont réparties dans les trois régions de la Méditerranée occidentale où les Phocéens ont été le plus actifs : au nord, le domaine marseillais; à l'est, la mer Tyrrhénienne, non loin d'Alalia; à l'ouest, la côte alicantine, où plusieurs petits comptoirs contrôlés par les Ibères étaient fréquentés par les Grecs de Marseille et d'Emporion. La conjonction est si remarquable qu'on a peine à croire qu'elle soit due au hasard.

Les îles de Lérins sont voisines de la colonie marseillaise d'Antipolis, dont huit kilomètres à vol d'oiseau les séparent. Les sources littéraires insistent fortement sur cette proximité ${ }^{41}$, qui se traduisait probablement, au moins à l'époque hellénistique, par une dépendance territoriale. À l'instar des îles Stœchades, les îles de Lérins devaient appartenir à Marseille, soit directement, soit indirectement à travers son apoikia d'Antipolis. Pianosa, en mer Tyrrhénienne, est située entre la colonie phocéenne d'Alalia et l'île d'Elbe, à michemin de la Corse et des ports du nord de l'Étrurie. Enfin, Tabarca fait face au port de Santa Pola, l'antique Alônis, où une

${ }^{39}$ Paulin de Nole donne, à la fin du IV ${ }^{\mathrm{e}}$ siècle de notre ère, une description précise de cet itinéraire. Le bateau pris par son ami Martinien s'écarte des côtes provençales à partir d'Arles, double la pointe de la Corse, puis, par l'île d'Elbe, gagne la région romaine (Carmina, 24). Sur ces questions, voir R. Dion, «Itinéraires maritimes occidentaux de l'Antiquité », Bull. Assoc. Géogr. fr., 243-244, 1954, p. 128-135.

40 Derrotero del Mediterráneo, op. cit., p. 285. La recommandation est ancienne : "On peut passer avec des vaisseaux et des galères entre le cap Saint Paul et cette isle [l'Isle Plane, c'est-à-dire Tabarca], rangeant un peu plus le cap que l'isle. Dans le milieu de ce passage, il y a cinq à six brasses d'eau " (Michelot, op. cit., p. 44).

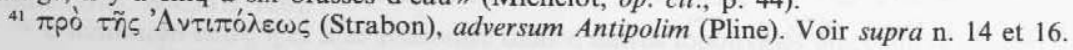


équipe d'archéologues franco-espagnole vient de ramener au jour les restes d'un petit établissement commercial fortifié, fondé vers 450 av. J.-C. et occupé jusqu'au troisième quart du siècle suivant ${ }^{42}$. Certes, ce modeste établissement ne peut pas être tenu pour une colonie de Marseille ${ }^{43}$, mais la complexité de ses défenses et la régularité modulaire de son plan témoignent d'une familiarité avec les modèles de l'architecture hellénique qui ne peut s'expliquer que par une fréquentation assidue, voire par une installation à demeure de commerçants grecs dans cette partie du littoral ibérique.

\section{Étymologie}

L'origine grecque du nom Planesia/Planasia ne fait aucun doute $^{44}$; dès le XVIII $^{\mathrm{e}}$ siècle, elle est reconnue par l'Espagnol Mayans, qui sera suivi par la plupart des philologues qui se sont penchés sur cette question. Néanmoins, un rapprochement inévitable avec le féminin de l'adjectif latin planarius ("plan», "de plain-pied $»)$, dont nous avons déjà fait état à propos des transformations du toponyme espagnol à l'époque médiévale et moderne, a fourvoyé beaucoup de bons esprits ${ }^{45}$. Il faut dire, à leur décharge, que les hésitations de Pline l'Ancien quant au nom et à l'identité de l'île tyrrhénienne, qui apparaît chez lui dédoublée en Planasia et en Planaria, et qui plus est assortie d'une étymologie confuse, n'ont fait qu'embrouiller la question.

Dans le livre III de l'Histoire naturelle, Pline mentionne une première fois l'île de Pianosa sous le nom de Planaria, en précisant qu'elle est «appelée ainsi à cause de son aspect, car elle s'étend à

${ }^{42}$ P. Moret, A. Puigcerver, P. Rouillard, M.J. Sánchez \& P. Sillières, «The Fortified Settlement of La Picola (Santa Pola, Alicante) and the Greek Influence in South-east Spain ", dans Social Complexity and the Development of Towns in Iberia (Proceedings of the British Academy, 86), Londres, 1995, p. 109-125. La publication d'ensemble des fouilles, qui se sont déroulées de 1991 à 1995, est en préparation.

${ }^{43} \mathrm{La}$ formule condensée d'Étienne de Byzance : "Alônis, île et ville de Marseille, d'après Artémidore" (s.v. 'A $\lambda \omega v i \iota_{\text {) }}$, ne doit pas être prise au pied de la lettre. La phrase d'Artémidore était probablement plus complexe, et il n'est pas exclu qu'Étienne en ait trahi le sens. S'il y eut un lien avec Marseille, il ne pouvait être que de nature économique.

${ }^{44} \mathrm{Il}$ est pourtant omis par P. Jacob dans sa liste des noms de lieu ibériques d'origine grecque, "Notes sur la toponymie grecque de la côte méditerranéenne de l'Espagne antique ", Ktema, 10, 1985, p. 247-271.

${ }^{45}$ Deux exemples parmi d'autres : "Le nom de cette île, dans Strabon, est Planasia, parce qu'en effet elle est très unie et sans hauteur " (abbé Courtépée, dans l'Encyclopédie de Diderot et d'Alembert, s.v. Lerina). Le nom de Planasia «est justifié par la nature et l'aspect de cette île» (E. Desjardins, Géographie historique et administrative de la Gaule romaine, I, Paris, 1876, p. 177). 
fleur d'eau et, par conséquent, risque de tromper les navires ${ }^{46}$. Quelques lignes plus loin, c'est la forme Planasia que donnent la plupart des manuscrits ${ }^{47}$, pour une seconde mention qui n'est assortie d'aucun commentaire. Les indications topographiques fournies par Pline dans les deux cas prouvent qu'il s'agit bien de la même île. La corruption du nom, dans le premier passage, peut être expliquée par l'influence conjuguée de trois faits de langue : d'abord, l'attraction de l'adjectif latin planarius; ensuite, le fait qu'en latin de très nombreux noms d'îles possèdent le suffixe -aria (dans le même paragraphe de Pline : Capraria, Menaria, Columbaria, Venaria); enfin, l'existence d'un suffixe adjectival italique -asio-, correspondant au latin -arius ${ }^{48}$, qu'on retrouve notamment dans la variante Caprasia du nom de l'île tyrrhénienne de Capraria $^{49}$. Le suffixe grec $-\alpha \sigma i \alpha$ de Planasia a pu être confondu par des locuteurs latins avec ce suffixe italique, autorisant une interprétation latine du mot et facilitant par là-même le passage à Planaria. Cette dérive sémantique remonte au moins à Pline luimême, puisque sa glose est en parfait accord avec le choix de la variante Planaria.

Cette glose, du reste, n'est pas exempte d'ambiguités. Fonctionnant en deux temps, elle développe d'abord planaria (aequalis freto: il s'agit bien d'une île "plane», plate et basse), puis, avec la formule navigiis fallax, elle introduit une notion non topographique qui nous oriente vers un tout autre registre sémantique, celui de l'île trompeuse. Or, nous allons bientôt voir que c'est précisément dans ce registre, celui de l'errance et d'une ambivalence inhérente à la nature même de l'île, que se meut le mot grec $\pi \lambda \alpha \nu \alpha \sigma i \alpha$. On a ainsi l'impression qu'à cette date - un moment indéterminé du $\mathrm{I}^{\mathrm{er}}$ siècle de notre ère - le sens originel de Planasia n'avait pas encore été complètement oblitéré par la réinterprétation latine du nom : Pline, à son insu, se fait l'écho de ce moment fugitif où un mot glisse insensiblement d'une langue et d'une signification dans une autre.

$\Pi \lambda \alpha \nu \eta \sigma i \alpha$ et $\Pi \lambda \alpha \nu \alpha \sigma i \alpha^{50}$ se rattachent à la famille de $\pi \lambda \alpha \nu \alpha \omega$

46 III 80 : LX p. a Corsica, Planaria a specie dicta, aequalis freto ideoque navigiis fallax. La tradition manuscrite (d'après l'édition Ian-Mayhoff, Teubner, 1905) présente presque unanimement la leçon Planaria (Planana R). Les éditeurs modernes ont adopté la correction Planasia.

${ }^{47}$ III 81 : Ilva (...) a Graecis Aethalia dicta. Ab ea Planasia XXVIII (Planaria $\mathrm{F}^{2}$ ).

${ }^{48}$ Cf. osc. sakrasias = lat. sacrariae (C.D. Buck, A Grammar of Oscan and Umbrian, Boston, 1904, p. 186).

49 Varron, Res rusticae, II 3, 3. On trouve aussi chez Pline un ostium Caprasiae, en Émilie (Hist. nat., III 120).

${ }^{50} L$ 'hésitation entre $\eta$ et $\alpha$ se rencontre dans d'autres toponymes grecs qui possèdent le 
( «faire errer», «égarer»), qui comporte à la fois des formes à sens

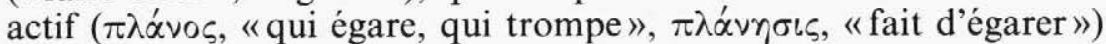
et des formes à sens passif, les plus nombreuses, qui gravitent

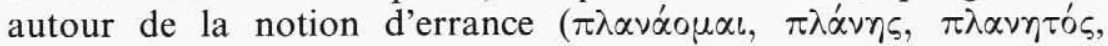
etc. $)^{51}$.

A partir de cette donnée incontestable, les interprétations divergent. J'écarterai d'emblée celles qui sont manifestement infondées. Pour P. Gössler, П $\lambda \alpha \nu \alpha \sigma i \alpha$ signifierait "éparpillé, dispersé», et s'appliquerait à un groupe d'îles ${ }^{52}$. Non seulement le sens proposé n'a qu'un lien des plus ténus avec la notion d'errance ou d'erreur, mais il est exclu que les noms Planasia et Planesia puissent s'appliquer à des archipels : dans tous les cas connus, il est bien établi qu'il s'agit d'une seule île. Schulten, pour sa part, voudrait que

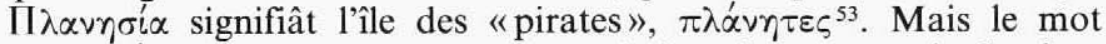
grec $\pi \lambda \alpha^{\prime} \nu \eta \varsigma$ n'a jamais eu ce sens, ni dans le passage de Strabon que Schulten invoque ${ }^{54}$, ni ailleurs : son horizon sémantique se borne à l'idée de vagabondage.

Plus sérieusement, la majorité des commentateurs se sont référés au verbe $\pi \lambda \alpha \nu \alpha \omega$ ou à l'adjectif $\pi \lambda \alpha^{\prime} \nu o s$, et — sans doute sous l'influence de Pline et de sa glose fallax - se sont prononcés pour un sens actif : "l'île qui trompe», «l'île qui égare» ${ }^{55}$. Rien de plus conforme à la topographie de ces îles, basses, donc peu visibles, et environnées de dangereux écueils. Mais on n'a pas vu

même suffixe. $\Upsilon \pi \varepsilon \rho \eta \sigma \tilde{\eta}$, ville d'Achaïe, est parfois nommée $\Upsilon \pi \varepsilon \rho \alpha \sigma i \alpha$ (Étienne de Byzance, s.v., qui relève aussi dans Théopompe l'ethnonyme $\Upsilon \pi \varepsilon p \alpha \sigma \varepsilon \varepsilon i \zeta \zeta$ à côté de l'ha-

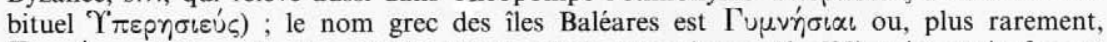

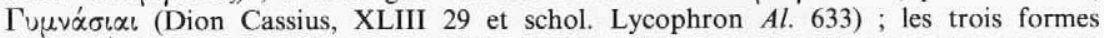
$\Lambda \varepsilon u x \alpha \sigma i \alpha$ (Denys d'Halicarnasse, I 53, cf. Pline, Hist. nat., III 85: Leucasia), Leucesia (dans le carmen Saliare cité par Scaurus, De orthographia, 28, 11) et $\Lambda \varepsilon u \varkappa \omega \sigma i \alpha$ (forme la plus courante) désignent la même île au sud du golfe de Salerne. Il est difficile de donner une explication satisfaisante à la coexistence des deux formes $\Pi \lambda \alpha v \eta \sigma^{\prime} \alpha$ et $\Pi \lambda \alpha \nu \alpha \sigma^{\prime} \dot{i} \alpha$ (chez Strabon notamment), compte tenu de la date tardive des premières attestations conservées - au ${ }^{\text {er }}$ siècle av. J.-C. - , des aléas de la transmission des textes et de l'attraction possible, en Étrurie, des suffixations locales en -asia et en -aria.

${ }^{51} \mathrm{P}$. Chantraine, Dictionnaire étymologique de la langue grecque. Histoire des mots, Paris, 1968, s.v. L'étymologie est obscure.

52 "Zerstreut liegende Inselgruppe» (dans $R E, \mathrm{XX}, 2$, 1950, s.v. Planasia).

${ }_{53}^{3}$ A. Schulten, Iberische Landeskunde, I, Strasbourg-Kehl, 1955, p. 249. Même opinion chez García y Bellido : «alude a los 'errantes' ( $\pi \lambda \alpha \dot{\alpha} \nu \tau \varepsilon \varsigma)$, o piratas que en ellas debían tener algún puesto" (Hispania Graeca, I, Barcelone, 1948, p. 140).

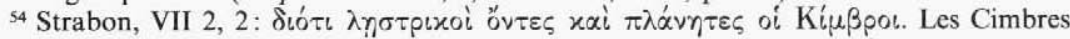
sont "des brigands et des vagabonds» dont il va sans dire que les méfaits ne se sont jamais exercés que sur la terre ferme.

${ }^{55}$ C'est l'interprétation traditionnelle, depuis le $\mathrm{XVIII}^{\mathrm{e}}$ siècle au moins : "Planasia es nombre griego. $\pi \lambda \alpha^{\alpha} v o \zeta$ en griego es el engañador, de aqui viene la voz $\pi \lambda \alpha v \alpha \sigma i \alpha$ » (Mayans, op. cit., p. 202). W. Pape et G. Benseler, Wörterbuch der Griechischen Eigennamen, Braunschweig, 1911, s.v., traduisent Planasia par Lügenfeld («terrain trompeur»). De même A. Solari, Topografia storica dell'Etruria, II, Pise, 1920, p. 173 : ingannatrice. 
que cette interprétation se heurtait à une sérieuse difficulté d'ordre morphologique. Planesia est en effet une formation adjectivale ${ }^{56}$ qui se rattache à une catégorie bien définie, celle des adjectifs à finale en $-\eta \sigma^{\prime} \alpha$. Cette finale dérive normalement d'un thème en -t-, qu'on retrouve en particulier dans les noms en - $\eta \varsigma$ et en - $\eta \tau \iota^{\prime} \varsigma^{57}$. La famille de $\pi \lambda \alpha \nu \alpha \omega$ possède les deux formes, $\pi \lambda \alpha^{\prime} \nu \eta{ }^{\prime}$ et $\pi \lambda \alpha \nu \eta$ -

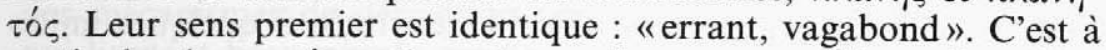
partir du champ sémantique de ces deux mots qu'il convient d'interpréter le toponyme, en écartant, par conséquent, l'éventualité d'un sens actif. Planesia n'est pas "l'île qui égare»; elle est "l'île de l'errance"».

\section{Insula Erroris}

On trouve dans l'Itinéraire maritime d'Antonin la mention fugitive d'une Insula Erroris, sise quelque part entre Carthagène et Césarée de Maurétanie ${ }^{58}$. Nom bizarre, sans exemple en toponymie latine, qui n'est sans doute que la maladroite adaptation d'un nom grec. On sait en effet, par de nombreux exemples, que le substantif error servait habituellement aux auteurs latins à traduire le grec $\pi \lambda \alpha \dot{\alpha} \nu$, "errance», "course errante», dans ses acceptions astronomiques aussi bien que maritimes ${ }^{59}$. Insula Erroris nous renvoie donc à la souche grecque de Planesia, tout en confirmant l'option étymologique que nous venons de prendre. On est même fort tenté de reconnaître là, dissimulée sous le masque latin, une quatrième Planesia. Le tour de la Méditerranée occidentale serait ainsi bouclé : après l'Italie, la Gaule et l'Espagne, l'Afrique.

Cette île de l'Errance n'est malheureusement pas identifiée avec certitude. On a pensé à l'île d'Alboran ${ }^{60}$, qui se trouve à peu près à mi-chemin entre Adra en Andalousie et Melilla sur la côte maro-

${ }^{56} \Pi \lambda \alpha \nu \eta \eta{ }^{\prime} \alpha$ n'a rien à voir, malgré les apparences, avec le substantif abstrait $\dot{\alpha} \pi \lambda \alpha \nu \eta \sigma \dot{\alpha} \alpha$ («infaillibilité»). Ce substantif à préfixe privatif est typiquement une formation savante, propre au vocabulaire philosophique; il n'est d'ailleurs attesté qu'à date tardive, vers 200 apr. J.-C. (Sextus Empiricus, Adversus mathematicos, VII 394).

57 Voir Chantraine, Formation des noms, p. 40-41 et 86 ; Buck \& Petersen, Reverse Index, p. 120.

58 Item inter Carthaginem Spartariam et Caesaream Mauretaniam insula Erroris et Tauria ; inter se habent stadia LXXV (p. 512 Wesseling; p. 250 Parthey-Pinder).

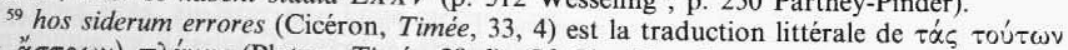
(= $\alpha \sigma \tau p \omega \nu) \pi \lambda \alpha^{\prime} \nu \alpha \varsigma$ (Platon, Timée, 39 d). Cf. Charisius Grammaticus, p. 451 Keil, 13. Error peut aussi désigner la course incontrôlée d'un navire (navium errorem, Bell. afr. VII 4) ou les errances d'un Ulysse ou d'un Énée (Virgile, AEn. VI 532 et VII 199, Cicéron, de off. I 113). ${ }^{60}$ C'est l'identification la plus courante jusqu'au $\mathrm{XIx}^{\mathrm{e}}$ siècle. Voir par ex. Morery,
Dictionnaire historique, s.v. Alboran. 
caine. Mais cette île est complètement isolée en haute mer, alors que l'Itinéraire annonce une distance de 75 stades seulement entre l'Insula Tauria et l'Insula Erroris. Comme d'autre part il n'existe aucune île en haute mer sur la route directe de Carthagène à Césarée de Maurétanie, force est de chercher ces deux îles près de la côte, un peu plus à l'ouest, c'est-à-dire dans les environs d'Oran ${ }^{61}$. On a donc proposé l'île Plane, située à quelques milles au large du cap Lindlès, à l'est de l'île Habibas (laquelle serait Tauria). Hypothèse séduisante, si l'on considère que le nom de l'île Plane pourrait conserver, comme celui de l'Isla Plana de Santa Pola, le souvenir d'une antique Planesia ${ }^{62}$.

\section{Errance des hommes, errance des îles?}

La notion d'errance, à laquelle nous nous sommes arrêté, demande à être précisée. La question est de savoir si la relation sémantique entre le nom $\vee \tilde{n} \sigma \circ \varsigma$ et l'adjectif devenu nom de lieu $\Pi \lambda \alpha \nu \eta \sigma^{\prime} \alpha$ doit être comprise comme une relation circonstancielle (l'île des errants) ou comme une relation attributive (l'île errante).

L'examen des autres noms de lieu grecs formés à l'aide du suffixe $-\eta \sigma^{\prime} \alpha$ invite à privilégier la première hypothèse. Comme les

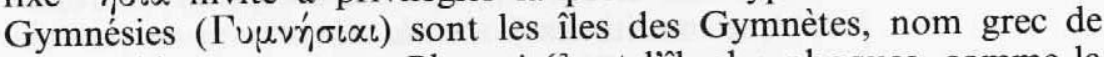
leurs habitants, comme Phocasia ${ }^{63}$ est l'île des phoques, comme la Magnésie $(M \alpha \gamma \nu \eta \sigma i \alpha)$ est la terre des Magnètes, ou comme Hypérésie ( $\left.{ }^{\prime} \Upsilon \tau \varepsilon \rho \eta \sigma \eta \eta\right)$ est la ville du héros Hypérès, Planesia serait l'île des errants, $\pi \lambda^{\prime} \alpha \nu \eta \tau \varepsilon \varsigma$. Ce sont évidemment des marins qu'il faudrait deviner sous ce qualificatif, des "vagabonds des mers",

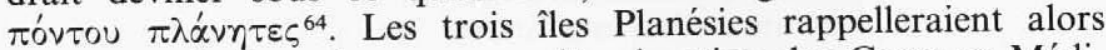
l'aventure des premiers voyages d'exploration des Grecs en Méditerranée occidentale, quand les navires progressaient d'île en île, au hasard des courants et des vents du Couchant. On peut aussi penser au prédécesseur illustre de ces marins grecs, à Ulysse, le vagabond par excellence ${ }^{65}$.

Malgré ces rapprochements suggestifs, il manque à cette interprétation la caution même indirecte des sources littéraires. On ne peut faire état d'aucun parallèle toponymique, d'aucun nom évo-

${ }^{61}$ Voir H. Treidler dans $R E$, XXVIII, 2, 1932, s.v. Tauria.

62 C. Müller, Claudii Ptolemaei Geographia, I (2), Paris, 1901, p. 593. S. Gsell, Atlas archéologique de l'Algérie, Alger et Paris, 1911, feuille 20 (Oran) et p. 1 du commentaire, se montre sceptique face à cette hypothèse qu'il ne rejette toutefois pas absolument.

${ }^{63}$ Pline, Hist. nat., IV 62.

${ }^{64}$ Tragica adespota, 100.

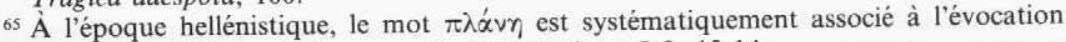
des «errances» d'Ulysse. Voir en particulier Strabon, I 2, 12-14. 
quant, par exemple, une "île des marins» ou une "île des égarés", qui nous prouverait que la figure du navigateur errant était admissible comme référent d'un nom d'île. C'est un point sur lequel on ne saurait assez insister : en grec ancien comme dans toute autre langue, la dénomination d'une terre nouvelle doit très peu à l'imagination et à la fantaisie des découvreurs; elle obéit, au contraire, à des mécanismes de dérivation très stricts et à des codes culturels qui limitent beaucoup le registre des noms disponibles. La toponymie des îles de la Méditerranée grecque se distingue, à cet égard, par son extrême conservatisme et par son caractère répétitif. Si l'on considère les noms d'îles assez peu nombreux qui sont réductibles à des noms communs grecs, on constate que les emprunts se cantonnent dans quatre domaines bien précis : la flore, la faune, la topographie (forme ou couleur de l'île) et la mythologie; de surcroît, ce sont souvent les mêmes noms que l'on retrouve d'un bout à l'autre de la Méditerranée ${ }^{66}$. Entre les «îles des pins", les "îles des serpents» et autres "îles des chèvres", il n'est pas sûr du tout qu'il y eût une place pour l'île des Errants.

Dans ce contexte, la seconde hypothèse - celle de l'île errante - m'a paru plus satisfaisante, parce qu'elle s'insère dans une série toponymique bien établie et, au-delà, dans une géographie mythique que jalonnent de nombreuses allusions littéraires : nous le verrons sans tarder. Encore fallait-il s'assurer que cette hypothèse était défendable d'un point de vue strictement linguistique. Deux faits m'en ont convaincu. À côté des exemples cités plus haut d'adjectifs en $-\eta \sigma^{\prime} \alpha$ qui équivalent à des compléments de nom indiquant une appartenance (l'île de ..., la terre de ...), on peut produire au moins un cas - mes recherches n'ont pas été exhaustives - de nom de lieu formé à l'aide du même suffixe, et cependant employé dans un sens attributif. Il s'agit de $\Lambda \varepsilon u \varkappa \omega \sigma i \alpha$, dite aussi $\Lambda$ sux $\alpha \sigma^{\prime} \alpha$ ou Leucesia $^{67}$, petite île qui ferme au sud le golfe de Paestum, dont le nom, formé sur la souche verbale $\lambda \varepsilon u \varkappa o ́ \omega$, signifie simplement «la Blanche».

Le second et le plus puissant argument en faveur d'une traduction par "île errante» nous attend en un lieu imprévu : le Curculio de Plaute. Dans cette comédie, Planesium est le nom d'une jeune esclave d'origine carienne, enlevée à sa famille alors qu'elle était

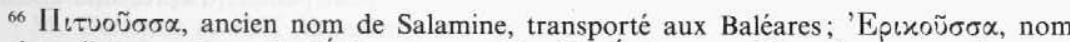

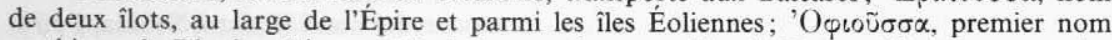
mythique de Rhodes, Ténos et Kythnos, porté par un îlot de Propontide et par une des

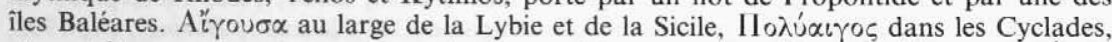

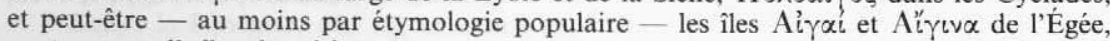
sont autant d'«îles des chèvres».

${ }^{67}$ La forme en -o- est la plus fréquente : $c f$. Strabon II 5, 19 et VI 1,1 et 6 ; Pline, Hist. nat., III 85. Pour les formes en -a- et en -e-, voir supra, n. 50. 
enfant et vendue à un leno d'Épidaure. Comme dans d'autres comédies de Plaute, les noms des personnages sont tous grecs et ont été empruntés à une pièce de la Comédie Nouvelle ${ }^{68}$. On serait donc en présence d'un nom П $\lambda \alpha \nu \dot{\gamma} \sigma \iota \nu$, non attesté par ailleurs, mais qui ne diffère de nos noms d'îles que par sa désinence. Sachant que les noms remplissent chez Plaute, comme dans ses modèles attiques, la fonction d'une clé de lecture, en rapport direct avec le caractère et l'histoire de chaque personnage ${ }^{69}$, il est aisé de décrypter celui de la jeune héroïne : c'est «l'errante». La révélation de l'identité de son père, à la fin de la pièce, vient confirmer, s'il était besoin, cette interprétation. Il s'appelle Peripla-

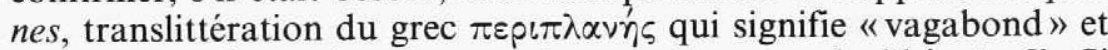
qui est formé sur la même racine que le nom de l'héroïne ${ }^{70}$. Si Planesium est la jeune errante, Planesia ne peut être que l'île errante.

\section{Îles mouvantes}

L'enracinement des îles au fond de la mer n'était pas, pour les Grecs, un fait d'évidence. Il existait une tradition cosmogonique, dont Homère, Pindare, Callimaque et quelques autres poètes nous ont gardé des échos, qui prêtait aux îles de la Méditerranée une ascendance marine. Nées de la mer, certaines d'entre elles étaient passées par un état ambigu, à mi-chemin de la matrice liquide indifférenciée et de la stabilité d'une terre accueillante aux humains : l'état d'île mouvante. Leur enracinement définitif sur le socle du monde habitable, leur annexion à l'oikoumène, était un des résultats de la victoire des Olympiens sur les forces dissolvantes du Chaos.

Pindare est l'interprète le plus suggestif de cette conception qui paraît si étrange à nos yeux, mais qui n'étonnait pas les Grecs. Les fragments conservés d'un hymne à Délos ${ }^{71}$ mêlent des métaphores apparemment contradictoires qui nous permettent, une fois rétabli l'ordre du récit mythique, de caractériser les deux phases successi-

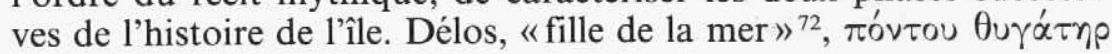

${ }^{68}$ Le modèle grec du Curculio est inconnu, mais on peut penser, d'après une allusion (v. 395), qu'il est antérieur au siège de Sicyone qui eut lieu en 303.

${ }^{69}$ G. Petrone, «Nomen / omen : poetica e funzione dei nomi (Plauto, Seneca, Petronio)", Materiali e Discussioni per l'analisi dei testi classici, 20-21, Pise, 1988, p. 33-70.

${ }^{70}$ Cf. K. Schmidt, "Die griechischen Personennamen bei Plautus», Hermes, 37, 1902, p. 377.

${ }^{71}$ Fr. 87-88 Schroeder.

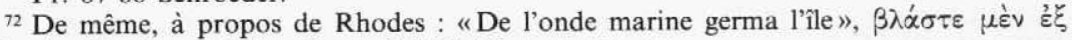

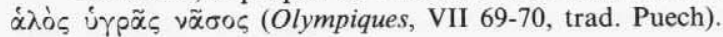


(v. 3), fut d'abord une île errante, "portée au gré des vagues",

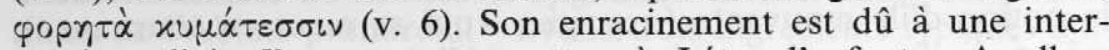
vention divine ${ }^{73}$ : pour permettre à Léto d'enfanter Apollon, quatre colonnes chaussées d'acier, suscitées du fond de la mer, donnèrent au roc mouvant l'assise de leurs chapiteaux (v. 8-11). On peut donc à bon droit la dire "fondée par les dieux», $\theta \varepsilon 0-$ $\delta \mu \alpha \tau_{\alpha}(v$. 1). Ce qui n'était auparavant qu'un roc stérile, $\pi \dot{\varepsilon} \tau p \alpha$,

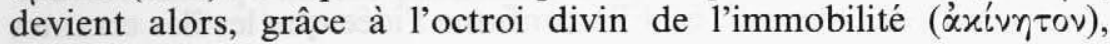
ferment de vie, ou pour reprendre l'audacieuse métaphore de Pin-

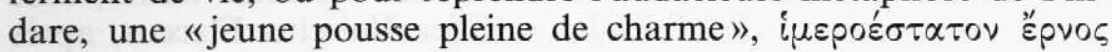
(v. 2-3).

La légende de Délos, primitivement flottante, est évoquée par d'autres sources plus tardives ${ }^{74}$, qui du point de vue qui nous occupe ici n'ajoutent rien à Pindare. Mais Délos n'est pas le seul cas d'île errante qu'aient connu les Grecs. Il existait, dans la géographie mythique de la Méditerranée archaïque, une série sans doute fort nombreuse d'îles réputées mouvantes; bien que les efforts de rationalisation des géographes hellénistiques et romains l'aient largement occultée, elle a survécu en partie dans la toponymie et dans des lambeaux de récits mythiques. Après que A.B. Cook eut, le premier, réuni le dossier littéraire des "Floating Islands ${ }^{75}$, plusieurs chercheurs ont insisté sur le caractère funeste de ces lieux ambivalents, marins et terrestres à la fois, qui signalaient des résurgences du chaos précosmogonique $^{76}$.

D'après une inscription, Patmos serait restée cachée dans les profondeurs de la mer jusqu'à ce qu'Oreste y ait établi le culte $\mathrm{d}^{\prime}$ Artémis ${ }^{77}$. Plus connue est. l'île "flottante» dans laquelle l'Odyssée place le séjour d'Éole ${ }^{78}$. Le même nom d' «îles flottantes», $\Pi \lambda \omega \tau \alpha i$ ṽ๊ $\sigma \circ \iota$, sera naturellement appliqué aux îles dites Éoliennes de la mer Tyrrhénienne ${ }^{79}$, mais c'est aussi le nom primitif des îles

\footnotetext{
${ }^{73}$ Pindare ne précise pas. D'autres sources désignent Zeus ou Poseidon ( $c f$. Ph. Bruneau, Recherches sur les cultes de Délos à l'époque hellénistique et à l'époque impériale, Paris, 1970 , p. 258 et 267 ).

${ }^{74}$ On en trouvera la liste dans A.B. Cook, Zeus, a Study in Ancient Religion, III, 2, Cambridge, 1940, p. $984-985$ et dans H. Gallet de Santerre, Délos primitive et archaïque, Paris, 1958 , p. 160.

${ }^{75}$ Cook, op. cit., p. 975-1015.

${ }^{76}$ M. Détienne et J.-P. Vernant, Les ruses de l'intelligence. La mètis des Grecs, Paris, 1974, p. 153-155 ; S. Vilatte, L'insularité dans la pensée grecque, Paris, 1991, p. 18 et 36.

77 Cook, op. cit., p. 985.

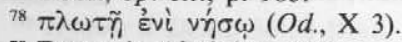

${ }^{79}$ Denys le Périégète, $465 \mathrm{sq}$, avec une explication peu convaincante : "parce qu'on peut naviguer tout autour $(\pi \varepsilon \rho i \pi \lambda \circ \varsigma)$ "), qu'Eustathe fait sienne à propos de l'île d'Éole de l'Odyssée. On préférera la lecture d'Aristarque (teste Eustathe ad $O d$. X 3), pour qui plôté signifie «mouvante» (
} 
Strophades ${ }^{80}$. Il est possible que ce dernier nom renvoie lui aussi à la notion d'île mouvante ( $\sigma \tau \rho \circ \varphi \alpha ́$, " "qui se meut en tournant»), même si les mythographes ont préféré une explication plus anecdotique : les îles Strophades seraient l'endroit où les Boréades, lancés à la poursuite des Harpyies, auraient fait demi-tour $\left(\sigma \tau \rho \circ \varphi \eta^{\prime}\right)^{81}$. Du reste, l'attestation d'un lien entre ces îles flottantes et les Harpyies, divinités néfastes, à la fois marines ${ }^{82}$ et infernales ${ }^{83}$, n'est pas sans intérêt. Elle confirme l'idée que les îles errantes ressortissent à la part la plus redoutable de l'élément marin, celle que dominent les forces du Chaos. Chez Valérius Flaccus, après qu'elles se sont arrêtées aux Strophades, les Harpyies exténuées implorent leur père Typhon qui se dresse aussitôt devant elles, "élevant avec lui les ténèbres, mêlant le haut et le bas»" ${ }^{84}$. Cette inquiétante apparition, dans laquelle les éléments se confondent et les repères spatiaux s'évanouissent, a précisément pour théâtre des îles errantes dont la nature hésite entre terre et mer, des îles qui désorientent parce qu'elles ne sont jamais là où on les a déjà vues.

On remarquera aussi que dans tous les cas que nous venons de citer, la condition d'île vagabonde est rejetée dans un temps révolu (Strophades, Patmos, Délos) ou dans l'irréalité du mythe (île d'Éole). On est dès lors en droit de se demander si ces témoignages épars ne gardent pas le souvenir d'un épisode cosmogonique oublié, appartenant à une tradition différente de celle dans laquelle Hésiode a puisé la matière de sa Théogonie. Cette supposition n'est pas tout à fait gratuite. On trouve en effet dans une scholie d'Apollonios de Rhodes une définition des îles errantes - jamais relevée à ma connaissance - qui fait peut-être allusion à un tel épisode. Parmi plusieurs explications possibles du fait qu'Apollonios ait appelé Plankte (c'est-à-dire, comme nous le verrons plus bas, "l'errante» ou "la flottante») l'île éolienne de Hiéra, le scholiaste propose incidemment celle-ci : "parce qu'autrefois toutes les îles étaient errantes et n'avaient pas de bases " ${ }^{85}$. Renvoi à un lointain passé, universalité du caractère erratique - donc chaotique - des îles primitives, opposition implicite entre les «bases» ferme-

\footnotetext{
${ }^{80}$ Apollonios de Rhodes, II 285 et 296-297; Valérius Flaccus, IV 512-513; Mela, II 110; Pline, Hist. nat., IV 55. Les Strophades sont deux îlots situés au sud de Zante.

${ }^{81}$ Apollonios de Rhodes, II 284-297. Les scholies ad loc. font remonter cette version du mythe à Antimaque.

${ }^{82}$ Maîtresses des tempêtes, on leur donnait pour père Poseidon ou Thaumas, lui-même fils d'Okeanos.

${ }^{83}$ D'autres traditions les reconnaissent filles de Typhon; elles sont aussi les messagères d'Hadès.

84 extulit adsurgens noctem pater imaque summis miscuit (Valérius Flaccus, IV 517). Selon Hygin, les îles Strophades étaient la demeure des Harpyies (Fabulae, XIV 18).

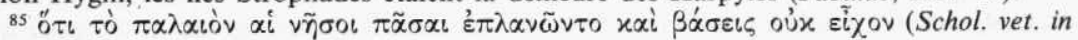
Apoll. Rhod., III 41-43).
} 
ment enracinées du kosmos et l'agitation incontrôlable des émanations de $\mathrm{l}^{\prime} \mathrm{A}$ bîme primordial ${ }^{86}$ : voilà trois ingrédients typiques d'un récit cosmogonique. Certes, on doit se garder de surinterpréter une phrase qui reste à la fois très vague et très isolée. Mais on voit mal quel motif autre qu'une réminiscence cosmogonique a pu inspirer au scholiaste une telle généralisation, alors que la question posée n'avait trait qu'au cas particulier d'une des îles Éoliennes.

\section{Îles et planètes}

Selon la légende, tant que dura sa course errante, Délos s'appela Asteria $^{87}$, l'île-étoile. Ce nom ne pouvait manquer d'inspirer les poètes. Tandis que Callimaque rappelle la course éperdue de la

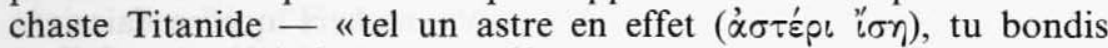
du haut du ciel dans le gouffre profond, pour fuir l'hymen de Zeus» ${ }^{88}$ - Pindare invoque l'île "que les mortels appellent Délos, et que les Bienheureux dans l'Olympe nomment l'astre, au

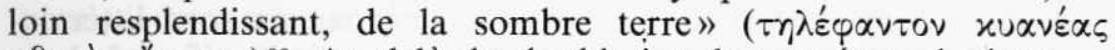
$\chi \theta 0$ vòs $\left.\chi^{\prime \prime} \sigma \tau \rho \circ v\right)^{89}$. Au-delà du double jeu de mot étymologique Dèlos la brillante, Asteria l'étoile -, c'est à un vertigineux jeu de miroirs que Pindare nous invite ici. Vue par les dieux du haut de l'Olympe, Délos-Astéria, brillante et vagabonde comme une étoile, laisse la même trace sur le fond bleu-noir de la mer qu'à nos yeux de mortels la révolution d'un astre errant dans le ciel nocturne.

L'analogie entre les déplacements réels des astres dans le ciel et les déplacements imaginaires des îles sans racines sur la vaste mer s'imposait donc naturellement à l'esprit d'un Grec. Trois noms d'îles en témoignent. Les deux premiers sont formés sur la racine aster qui désignait en grec toutes sortes de corps célestes (planètes, étoiles fixes, étoiles filantes) : Asteria, dont nous venons de parler, et Asteris, nom d'un îlot voisin d'Ithaque ${ }^{90}$. Cette Asteris n'est, comme Délos, qu'un aride rocher ${ }^{91}$; corpuscules minéraux égarés

${ }^{86}$ Sur cette opposition entre un abîme sans fond et un monde aux fermes assises, $c f$. Hésiode, Théogonie, 116-128 et 724-745.

${ }^{87}$ Pindare, Péan V, 42 ; Callimaque, Hymne à Délos, 36-38; Hygin, Fabulae, 53. Cf. Wernicke dans RE, II (2), 1896, s.v., et H. Papastavrou dans LIMC, II (1), 1984, p. 903 sq. Astéria est une Titanide, sœur de Léto ; selon une partie de la tradition, elle s'est transformée en caille (ortyx) et jetée dans la mer pour échapper aux ardeurs de Zeus; elle devient alors un rocher flottant qui prend le nom d'Ortygie et qui, une fois fixé, s'appellera Délos.

${ }^{88}$ Hymne à Délos, 36-38 (trad. Cahen).

${ }^{89}$ Fr. 87-88 Schroeder, 4-5 (trad. Puech).

${ }^{9}$ Odyssée, IV 844-846.

${ }^{91}$ Asteris est décrite par Homère comme une île rocheuse $(\pi \varepsilon \tau p \dot{n} \varepsilon \sigma \sigma \alpha, 844)$ et de petite dimension (oủ $\mu \varepsilon \gamma \alpha \dot{\alpha} \lambda \eta, 846$ ). 
dans la mer, toutes deux se prêtaient à merveille à la métaphore astrale.

Le troisième nom est Planesia, qui est très exactement aux "planètes", $\pi \lambda^{\prime} \alpha \nu \eta \tau \varepsilon \varsigma^{92}$, ce qu'Asteria est à aster. Dans les deux cas, une même souche lexicale a servi à désigner l'astre errant dans le ciel et - sous une forme dérivée - l'île errant sur la mer. Étonnante symétrie des bifurcations sémantiques, qui nous confirme dans notre interprétation du nom Planesia, et qui s'explique peut-être par l'existence d'un référent mythique commun.

\section{Encore des roches mouvantes : les Planctes}

La légende des Argonautes présente sous le nom de «roches Planctes" deux falaises ou deux hauts rochers qui se faisaient face et qui, doués de mouvement, étaient capables de se refermer sur les vaisseaux imprudemment engagés entre leurs parois ${ }^{93}$. Leurs affinités avec les îles errantes ont été signalées de longue date ${ }^{94}$. Le

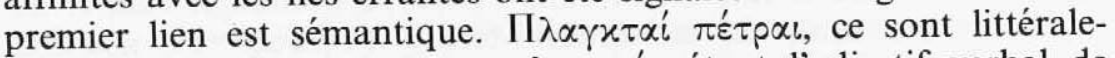
ment les «roches errantes», $\pi \lambda \alpha \gamma x \tau o ́ s$ étant l'adjectif verbal de $\pi \lambda \alpha^{\prime} \zeta \omega$, qui au passif signifie "errer» ou, plus spécifiquement, «flotter au hasard». Ainsi, dans l'hymne à Délos de Callimaque, l'errance d'Astéria est d'abord décrite à l'aide du participe passif

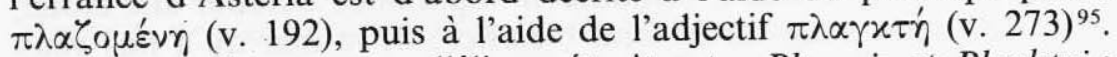
On observe donc un parallélisme étroit entre Planesia et Planktai : leur racine verbale a la même signification, "errer», et leur emploi est réservé exclusivement (Planesia) ou préférentiellement (Planktai) au domaine maritime. Le rapprochement est encore plus troublant si l'on considère que le participe $\pi \lambda \alpha \zeta^{\prime}{ }^{\prime} \mu \varepsilon v o \varsigma$ a aussi servi à

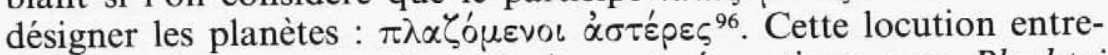
tient le même rapport étymologique et sémantique avec Planktai que $\pi \lambda^{\prime} \alpha \nu \eta \tau \varepsilon \varsigma$ $\alpha \sigma \tau \varepsilon^{\prime} p \varepsilon \varsigma$ avec Planesia. C'est tout naturellement que la Souda entérine ces multiples convergences lexicales, en définissant $\Pi \lambda \alpha \gamma \gamma \tau \dot{\eta}$ (s.v.) comme $\dot{\eta} \pi \lambda \alpha \nu \omega \mu \dot{\varepsilon} v \eta$.

Le second lien est géographique. Si, pour Hérodote (IV 85), les

${ }^{92}$ Le mot grec s'applique génériquement à tous les astres erratiques, y compris les comètes (lat. errantia sidera).

${ }_{93}$ Leur première mention se trouve dans l'Odyssée, XII 59-72 et XXIII 327.

${ }_{94}$ Cook, op. cit., p. 975-978, repris et développé par J. Lindsay, The Clashing Rocks, a Study of early Greek Religion and Culture and the Origins of Drama, Londres, 1965, passim. La notice de F. Gisinger dans $R E$, XX, 2, 1950, col. 2187-2199, rassemble l'essentiel du dossier littéraire.

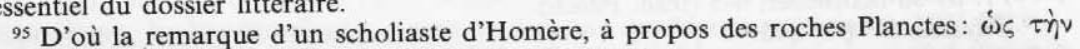

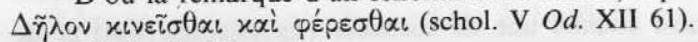

96 Timée, fr. 97 a. 
roches Planctes se confondent avec les roches Cyanées du Bosphore, la tradition hellénistique, à la suite de Timée et de Pisistrate de Lipara ${ }^{97}$, est unanime à situer les roches Planctes à proximité du détroit de Messine. Certains auteurs, évoqués par Strabon, avaient même proposé de placer les roches Planctes au détroit de Gibral$\operatorname{tar}^{98}$. Les Planctes appartiennent donc, comme les îles Planésies, à la Méditerranée occidentale. Mais il y a plus. Avant de se risquer entre les falaises mouvantes des Planctes, les Argonautes sont passés, dans la variante du mythe qui leur fait prendre une route occidentale au retour de leur expédition, par les îles Stœchades, «où des autels et des rites furent établis en leur honneur pour toujours", et par Aithalia où "ils essuyèrent avec des galets leur abondante sueur $"{ }^{99}$. Les îles Planésies de Provence et de Toscane se trouvent donc à proximité de deux escales légendaires du navire Argo : les îles d'Hyères et l'île d'Elbe.

Il va sans dire qu'aucune de nos trois Planésies ne présente les traits effrayants d'une roche Plancte. Tabarca et Pianosa sont des îles isolées, ce qui interdit d'emblée tout rapprochement. Mais on peut se demander si la disposition des îles de Lérins, séparées par une passe qui, quoique navigable ${ }^{100}$, est réputée dangereuse en raison des courants qui s'y engouffrent ${ }^{101}$, n'était pas propre à susciter l'image des rochers naufrageurs. Après tout, si les habitants des îles d'Hyères revendiquaient l'honneur d'avoir accueilli les Argonautes sur leurs rivages, pourquoi ceux des îles de Lérins ne se seraient-ils pas vantés d'avoir mis le navire Argo en péril? Cette hypothèse soulève cependant bien des difficultés. Elle ne permet pas d'expliquer le fait que seule l'île méridionale ait porté le nom d'île errante. De plus, les îles de Lérins sont basses, boisées et habitables, soit tout le contraire des falaises nues et hostiles qu'on prête aux Planctes de la légende.

D'un point de vue plus général, la pertinence d'un rapprochement entre Planktai et Planesiai, entre îles errantes et roches mouvantes, n'est même pas assurée. L'analogie des noms et l'attribut commun de la mobilité masquent en fait des natures essentiellement distinctes. Les Planctes ne sont pas des îles, mais deux

97 Schol. vet. in Apoll. Rhod. IV 786.

98 "Certains transportent ici [au détroit de Gibraltar] les Planctes et les Symplégades, qu'ils identifient avec les Colonnes» (Strabon, III 5, 5).

${ }_{99}$ Apollonios de Rhodes, IV, respectivement v. 650-652 et 654-657 (trad. Vian).

100 "On peut passer, dans une nécessité, entre ces deux isles avec une galère, y ayant au moins deux brasses de profondeur» (Michelot, op. cit., p. 187).

101 «Le passage entre les deux îles est encombré par le plateau du Milieu. (...) Par mistral ce mouillage [sur le plateau du Milieu] est intenable, la mer se formant très rapidement et les courants devenant violents" (Instructions nautiques, série D, II, Côtes sud de la France, Paris, 1968, p. 189). 
rochers formant couple, situés chacun à la pointe d'une péninsule ou d'une grande île. Leur mouvement, à la fois vertical et horizontal, est, en dépit de sa violence, régulier et cyclique ${ }^{102}$. Dans le sens horizontal, loin d'être une errance aléatoire, il s'apparente à la mécanique d'une paire de mâchoires qui s'ouvrent et se referment. Dans le sens vertical, il suppose une sorte d'enracinement alternatif, dans le fond de la mer puis au plus haut du ciel ${ }^{103}$. La verticalité est d'ailleurs la composante essentielle des Planctes - la seule sur laquelle Homère s'étende un peu ${ }^{104}$ _, alors qu'elle n'intervient jamais dans les descriptions connues d'îles errantes ou flottantes.

Ajoutons, sous le rapport de l'étymologie, que le glissement sémantique de $\pi \lambda \alpha^{\prime} \zeta o \mu \alpha \iota$ et de $\pi \lambda \alpha \gamma x \tau \tau^{\prime} \varsigma$ vers la notion d'errance est peut-être relativement tardif. Appliqué aux vagues de la mer, $\pi \lambda \alpha^{\prime} \zeta \omega$ devait avoir initialement le sens de "frapper» ( $c f . \pi \lambda \dot{\eta} \sigma \sigma \omega$ et lat. plango $)^{105}$, ce qui s'accorderait mieux avec les descriptions traditionnelles des roches Planctes, qui sont présentées avant tout comme des roches fracassantes et entre-choquantes ${ }^{106}$.

Que retenir de ce bref examen? Les roches Planctes sont, à l'origine, irréductibles au thème des îles errantes; néanmoins, une assimilation partielle a été rendue possible par le sémantisme convergent de leurs noms et par la similitude de leur environnement mythique. Il est en tout cas peu probable qu'elles aient contribué à la formation d'une représentation mythique des îles Planésies.

\section{Héraclès à Lérins?}

A plusieurs reprises dans les pages qui précèdent, j'ai été amené à supposer que la figure de l'île errante s'insérait dans une construction mythique dont l'épisode essentiel était l'intervention d'un dieu ayant le pouvoir d'immobiliser et d'enraciner l'île. Spéculation toute théorique, dans le cas de Tabarca et de Pianosa, mais qui s'appuie à Lérins sur plusieurs indices qui m'ont paru dignes de

${ }^{102}$ Les descriptions les plus explicites sont celles d'Apollonios de Rhodes, II 553-618 et IV 942 sqq, et de Valérius Flaccus, Argonautica, IV 657-660.

${ }^{103} \mathrm{Cf}$. Apollonios de Rhodes, IV 944-947 : "Tantôt les rocs, semblables à des falaises, allaient toucher le ciel (

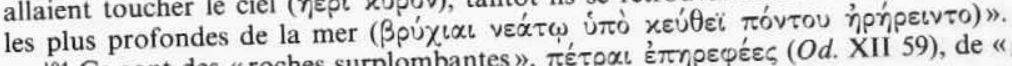

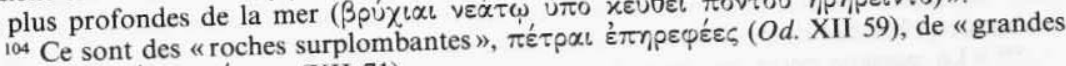
roches ", $\mu \varepsilon \gamma^{\prime} \alpha \lambda \alpha_{l} \pi \dot{\varepsilon} \tau \rho \alpha_{l}$ (XII 71).

${ }^{105}$ Chantraine, Dictionnaire étymologique, s.v.

$106 \mathrm{Ce}$ dernier adjectif traduit exactement le nom des roches Symplegades du Bosphore, qui sont décrites dans les mêmes termes que les Planctes. 
considération. Je prendrai donc le risque, avant de clore cette enquête, de m'avancer sur le terrain mal assuré de la légende, en centrant désormais mon attention sur l'île Saint-Honorat.

\section{Saint Honorat et les serpents}

La vie de saint Honorat, composée par saint Hilaire en 430, un an après la mort du fondateur de l'abbaye de Lérins ${ }^{107}$, contient plusieurs traits légendaires qui détonent singulièrement dans le cours d'un récit édifiant qui ne montre par ailleurs aucune propension au merveilleux. L'arrivée du saint sur la petite île de Lérins est décrite en ces termes : "Il se rendit sur une île qui était déserte en raison d'un sol excessivement aride, et à laquelle on n'abordait même pas de peur des animaux venimeux qui l'infestaient». Mais devant lui, «l'horreur du désert s'évanouit, les serpents grouillants disparaissent»108. "Taries depuis des siècles, les eaux de source s'écoulent en abondance (...); l'eau douce court se mêler aux flots salés de la mer ${ }^{109}$. Saint Honorat a obtenu de Dieu trois miracles : il a chassé les serpents, a fait jaillir les sources et, comme l'indique la suite du récit, a fait donner les plus beaux fruits à une terre qui jusqu'alors n'était qu'asperitas et squalor.

$\mathrm{La}$ Vida de San Honorat du troubadour niçois Raymond Féraud, écrite au XIII ${ }^{\mathrm{e}}$ siècle, présente autrement le même épisode légendaire. Ce n'est plus une foule de reptiles venimeux qui interdisent le séjour des îles de Lérins, mais un couple de serpents monstrueux bien individualisés, qui portent même un nom : Léry et Rins ${ }^{110}$. J. Coupry et G. Vindry ont proposé de reconnaître dans ces deux démons-serpents les divinités topiques et éponymes des îles, Lérô et Lériné. Cette identification suppose que la légende médiévale est venue se greffer, en l'altérant à peine, sur un mythe préchrétien. Lérô et Lériné, dont nous ne connaissions que les noms ${ }^{111}$, s'affirmeraient alors comme des créatures chtoniennes, comparables à cette Echidna, sœur de Géryon, dont Hésiode a décrit la tête de femme séduisante et la hideuse queue de serpent ${ }^{112}$,

107 Sermo de vita sancti Honorati (Patrologie, L, 1859, col. 1249-1272).

${ }^{108}$ III, I5: Vacantem itaque insulam ob nimietatem squaloris, et inaccessam venenatorum animalium metu, petit. (...) Fugit horror solitudinis, cedit turba serpentium.

109 III, 17 : Negatae a saeculis aquae largiter fluunt, (...) in media maris amaritudine dulces profluebant.

${ }^{110}$ D'après Coupry et Vindry, op. cit. (supra n. 13), p. 358.

111 Ces noms ne sont pas grecs comme Planasia ; leur origine a toute chance d'être locale et pré-indo-européenne (Ch. Rostaing, Essai sur la toponymie de la Provence, Paris, 1947 , p. $189-190$ ).

112 Théogonie, 295 sqq. 
comparables surtout au serpent fabuleux des Hespérides ${ }^{113}$ qu'Héraclès devra tuer pour s'emparer des pommes d'or.

Or, le souvenir d'un Héraclès tueur de serpents n'était pas tout à fait éteint dans la Provence du haut Moyen-Âge, s'il faut en croire un obscur chroniqueur $\mathrm{du}^{\mathrm{e}}$ siècle, un certain Galfredus ${ }^{114}$, qui nous montre Hercule tuant un prodigieux serpent dans l'île de Mésé, l'une des Stœchades dont Pline nous a laissé le nom ${ }^{115}$. Certes, cet exploit non homologué n'est peut-être qu'une fabrication tardive, forgée de toutes pièces dans une cellule monacale. Mais les analogies avec la légende de Lérins - une île marseillaise pour théâtre, un reptile monstrueux pour adversaire - ont de quoi intriguer. L'indice est sans doute trop ténu pour nous permettre d'affirmer qu'Héraclès se cache derrière saint Honorat, et que Lérô et Lériné sont, comme Géryon et Éryx ${ }^{116}$, de ces créatures chtoniennes, maîtresses du sol, que le héros thébain a dû vaincre pour ouvrir aux Grecs les portes de l'Occident; il est cependant assez suggestif pour nous inciter à pousser plus loin l'enquête dans le vaste domaine du mythe héracléen.

\section{Héraclès d'île en île}

On évoque souvent l'itinéraire terrestre d'Héraclès vers le pays de Géryon et des Hespérides; l'épisode des cailloux de la Crau et le nom de la voie Héracléenne en sont les jalons les plus connus. Mais une tradition concurrente faisait d'Héraclès un navigateur expérimenté117 : c'est par mer qu'il aurait gagné l'Occident et qu'il en serait revenu. Apollodore nous le montre faisant voile d'Érythie vers Tartessos ${ }^{118}$, puis reprenant la mer en direction de la Ligurie et de l'Italie. La toponymie insulaire marque les étapes de cette navigation. On compte ainsi au moins deux "îles d'Héraclès». sur les côtes méridionales de l'Espagne ${ }^{119}$, une autre à la

113 Dragon selon Apollodore (Bibliotheca, II 113), serpent selon Hésiode (Théogonie, 333-336) et Phérécyde (Schol. Apoll. Rhod. IV 1396).

114 Cité par J. Coupry, "Catalogue (...) des dieux et héros helléniques à Marseille», dans Marseille grecque et la Gaule (Études massaliètes, 3), Aix-en-Provence, 1992, p. 159, à qui l'importance de cette réminiscence mythique n'avait pas échappé.

${ }_{115}$ Hist. nat., III 79. C'est sans doute l'actuelle Porquerolles.

116 Apollodore, Bibliotheca, II 106-109.

${ }^{117}$ L. Lacroix a justement insisté sur la place importante qu'occupait la mer dans la geste d'Héraclès, malgré les silences de la tradition littéraire («Héraclès, héros voyageur et civilisateur ", Bull. Lettres et Sciences mor. et pol. de l'Acad. r. de Belgique, 60, 1974, p. 3459).

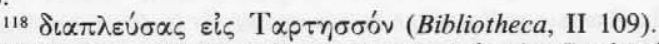

${ }^{119}$ Strabon, III 4, 6 (Escombrera, près de Carthagène) et III 5,5 (Saltes, près de

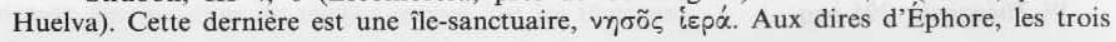


pointe nord-ouest de la Sardaigne ${ }^{120}$, une autre encore parmi les îles Lipari ${ }^{121}$; et le nom des Baléares viendrait, selon une des deux versions enregistrées par Tite-Live, de Balius, "un compagnon d'Héraclès abandonné là alors qu'Héraclès naviguait vers le pays de Géryon ${ }^{122}$. De nombreux ports revendiquaient une escale du héros, et plusieurs d'entre eux, en Italie, lui devaient même leur nom. On retiendra surtout que deux de ces ports héracléens encadraient la Planésie ligure : Heraclea Caccabaria ${ }^{123}$ à l'ouest (probablement à Cavalaire ${ }^{124}$ ), Monaco et son sanctuaire d'Héraclès monoikos $^{125}$ à l'est.

C'est donc à cet Héraclès navigateur, explorateur et civilisateur de l'Extrême Occident, qu'une tradition locale peut avoir opposé les dieux-serpents de Lérins. Mais parler d'un Héraclès navigateur et civilisateur revient nécessairement à évoquer la figure de Melqart. Les remarquables travaux de Corinne Bonnet ont en effet montré qu'il s'était tissé, dès l'époque orientalisante, un dense réseau de correspondances, d'assimilations et de contaminations entre le Phénicien Melqart et le Grec Héraclès ${ }^{126}$. Ce phénomène d'assimilation, ébauché à Chypre $\mathrm{au}_{\mathrm{vI}}^{\mathrm{e}}$ siècle, voire dès le $\mathrm{VII}^{\mathrm{e}}$ siècle ${ }^{127}$, s'affirme à Thasos et à Tyr dans la première moitié $d u v^{e}$ siècle $^{128}$. Et c'est précisément à Tyr que s'élabore un mythe de fondation, lié à Héraclès-Melqart, dont plusieurs détails rappellent l'épisode légendaire de Lérins. Qu'on ne se méprenne pas sur la portée de cette comparaison : il ne s'agit pas d'un détour hasardeux par la mythologie proche-orientale. Le mythe que nous allons évoquer doit au moins autant aux Grecs qu'aux Phéniciens; il est le fruit "d'une recomposition sur le mode grec d'un Melquart contaminé par son équivalent grec ${ }^{129}$.

îlots du Cap Sacré, à l'extrême pointe de l'Europe, étaient eux aussi consacrés à Héraclès (assertion réfutée par Artémidore d'après Strabon, III 1, 4).

${ }^{120}$ Pline, Hist. nat., III 84.

${ }^{121}$ Heraclea (Mela, II 120) ou Herculis insula (Tab. Peut.).

${ }^{122}$ Tite-Live, Periochae, 60 : Baleares a teli missu appellati aut a Balio, Herculis comite ibi relicto, cum Hercules ad Geryonen navigaret.

${ }^{123}$ Itinéraire maritime, p. 505 Wesseling.

124 J.-P. Brun, "Le village massaliote de La Galère à Porquerolles", dans Marseille grecque et la Gaule (Études massaliètes, 3), Aix-en-Provence, 1992, p. 286.

${ }^{125}$ Strabon, IV 6, 3 : «Le Port de Monoikos (...) possède un sanctuaire d'Héraclès dit Monoikos ».

${ }^{126}$ C. Bonnet, Melqart. Cultes et mythes de l'Héraclès tyrien en Méditerranée (Studia Phoenicia, VIII), Louvain, 1988. Voir aussi C. Jourdain-Annequin, Héraclès aux portes du soir. Mythe et histoire, Besançon, 1989.

${ }^{127}$ Bonnet, op. cit., p. 401 et 410-414; Jourdain-Annequin, op. cit., p. 167-169.

${ }^{128}$ Hérodote, II 44 et Pausanias, V 25, 12, textes commentés par Bonnet, op. cit., p. 47

50 et $346-354$.

${ }^{129}$ Bonnet, op. cit., p. 98 . 


\section{Melqart et l'enracinement de Tyr}

Le mythe de fondation de Tyr nous a été conservé par Nonnos ${ }^{130}$. A Dionysos qui lui demande "quel dieu a fondé cette cité, (...) qui a élevé ces rochers et les a enracinés dans la mer» (v. 423-425), Héraclès explique comment il a appris aux hommes à construire le premier bateau et les a conduits en un lieu "où deux rochers instables et vagabonds flottent sur la mer ${ }^{131}$. Ce sont les Roches Ambrosiennes; un olivier pousse sur elles, qui porte une coupe et un aigle à son sommet; un serpent est enroulé autour de son tronc. Les hommes devront sacrifier l'aigle «sur

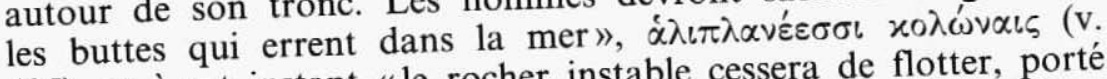
$495)$, et à cet instant "le rocher instable cessera de flotter, porté au hasard par les flots; ceinturé par des fondations inébranlables, il se fixera à la roche vierge ${ }^{132}$. Verbeux comme à son habitude, Nonnos use de cinq adjectifs différents pour qualifier l'errance des roches primitives; on notera que l'un d'entre eux, le composé $\dot{\alpha} \lambda \iota \pi \lambda \alpha \nu \eta \dot{\zeta}$, , "qui erre sur la mer", est formé sur la même racine que Planesia.

Le récit de Nonnos est complété par plusieurs représentations figurées sur des monnaies tyriennes du $\mathrm{III}^{\mathrm{e}}$ siècle de notre ère ${ }^{133}$. On y voit diversement associés, selon les types monétaires, trois éléments récurrents : les deux roches du mythe de fondation (sous l'aspect de deux stèles jumelles), l'olivier sacré et une source émanant de la base des stèles ${ }^{134}$. La source n'est pas mentionnée par Nonnos, mais elle est indispensable à la bonne intelligence du mythe : fixer des roches jusque-là errantes et stériles, en faire jaillir une source d'eau douce, c'es les transformer en île habitable, c'est permettre aux hommes de s'y établir et d'y prospérer.

Il est à peine nécessaire d'insister sur le parallélisme des schémas mythiques relevés à Lérins et à Tyr. Vainqueur des serpents ${ }^{135}$, sourcier et fertilisateur sur une île dont le nom rappelle la primitive errance, le saint Honorat de la légende se profile comme le calque

${ }^{130}$ Dionysiaca, XL 423-500.

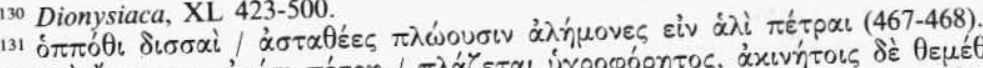

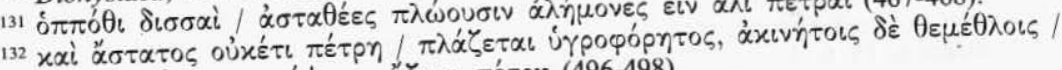

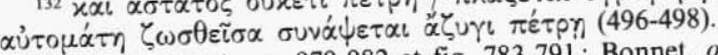

${ }^{133}$ Cook, op. cit., p. 979-982 et fig. 783-791; Bonnet, op. cit., p. 87-90.

${ }^{134}$ Le serpent, absent dans cette série, apparaît sur d'autres monnaies tyriennes lové autour d'une pierre ovoïde dont la signification n'est pas clairement établie.

${ }^{135}$ Le thème de la lutte contre le serpent a été l'un des vecteurs du rapprochement entre Héraclès et Melqart. C'est ce qu'indique notamment la découverte, sur le site phénicien de Motyé, d'une terre cuite de fabrication locale, de Lerne (Bonnet, op. cit., av. J.-C., qui représente le combat d'Heracles contre p. 276). 
presque ${ }^{136}$ exact du fondateur de Tyr. Il n'est pas jusqu'à la dualité des «rochers vagabonds» qui ne soit rappelée à Lérins par l'existence de deux îles presque jumelles.

\section{Circulations souterraines}

On trouve dans le roman d'Achille Tatius de curieux détails sur le régime des eaux souterraines de l'île de Tyr, devenue presqu'île après le siège d'Alexandre. "Tyr n'est pas enracinée dans la mer : l'eau coule en dessous, par un passage situé sous l'isthme. D'où le spectacle étrange d'une ville dans la mer et d'une île dans la terre ${ }^{137}$. Remarquons en passant, derrière l'antithèse trop facile, cette image d'un lieu hybride où la terre et la mer cessent de se départager. Elle revient comme un leit-motiv chaque fois qu'il est question d'îles errantes; nous l'avons déjà rencontrée à propos des Strophades et de Planasia-Pianosa, l'île qui se confond avec la mer. Mais l'intérêt principal de ce texte est ailleurs. En recueillant une tradition locale, selon laquelle le sous-sol de Tyr était parcouru de fissures par lesquelles la mer circulait librement, il nous apprend que l'enracinement de leur île n'était pas considéré par les habitants de Tyr comme un fait définitivement acquis. L'œuvre de Melqart était toujours à refaire, et l'on peut même penser que l'un des enjeux du culte du dieu poliade était cette perpétuelle reconduction de la victoire des hommes, aidés par le dieu civilisateur, sur les éléments destructeurs ${ }^{138}$.

Ces infiltrations souterraines, ces interpénétrations d'éléments qui s'excluent normalement, nous les retrouvons sur deux sites fameux de l'Occident phénicien, tous deux consacrés à Melqart : Gadès et Lixus. À Gadès, aux grandes marées du solstice d'été, l'eau recouvrait le soubassement du temple d'Héraclès-Melqart ${ }^{139}$ bâti sur l'île-sanctuaire de Kotinoussa; et l'on trouvait dans ce même sanctuaire une fontaine où le régime des eaux variait en fonction du flux et du reflux des marées ${ }^{140}$. L'eau de mer circulait donc au-dessus et au-dessous de l'île. Près de Lixus, dans le golfe Emporique, il existait un antre que la marée haute envahissait régulièrement; et c'est devant cet antre, à l'abri de la marée, que

${ }^{136}$ L'aigle et la coupe manquent à Lérins, mais on remarquera qu'ils sont également omis sur les monnaies tyriennes où figurent l'arbre, les deux stèles et la source.

${ }_{137}$ Achille Tatius, Leucippe et Clitophon, II 14, 4.

${ }^{138} \mathrm{La}$ vulnérabilité de Tyr face aux éléments était presque passée en proverbe : Tyros et ipsa tam mouetur quam diluitur, "Tyr est autant secouée [par les séismes] que rongée [par la mer]" (Sénèque, Naturales Quaestiones, VI 26, 4).

${ }^{139}$ Strabon, III 5, 9 (d'après Posidonios).

140 Strabon, III 5, 7 (d'après Polybe). 
se trouvait l'autel d'Héraclès ${ }^{141}$. Ces anecdotes et ces légendes renvoient peut-être, en dernier ressort, à une même conception mythico-géographique qui mettait l'accent sur la fusion originelle des éléments et sur la précarité des sites insulaires, dont l'ancrage n'était jamais tout à fait maîtrisé par un dieu tutélaire en lutte constante avec les puissances infernales.

Parallèlement, on constate que le culte d'Héraclès-Melqart prend à Gadès et à Lixus des formes qui rappellent à la fois le mythe de Tyr et la légende de Lérins. À Gadès, le sanctuaire de Melqart, sur l'îlot de Kotinoussa, contenait deux stèles et un olivier d'or ${ }^{142}$ qui se référaient manifestement au mythe fondateur de la métropole tyrienne, tandis que "l'homme de la mer», un monstre apode de taille gigantesque qu'une étrange anecdote nous montre foudroyé par le dieu - en effigie probablement, et dans le cadre d'un rite sur le rivage de l'île ${ }^{143}$, fait inévitablement penser aux adversaires anguipèdes de l'Héraclès grec. À Lixus, l'autel d'Héraclès se dressait sur un îlot au milieu de l'estuaire du Loukkos, environné de méandres qui figuraient, selon Pline, les replis du serpent des Hespérides, non loin du verger aux arbres fabuleux ${ }^{144}$. Ce sont donc les mêmes éléments mythiques - l'île errante ou infiltrée par la mer, les deux roches, l'arbre sacré, le serpent ou le monstre apode, la source - qui se retrouvent d'un site à l'autre sur les étapes occidentales de la route d'Héraclès-Melqart. Le tableau suivant permet d'apprécier l'ampleur de ces correspondances.

\begin{tabular}{|l|l|l|l|l|l|}
\hline Tyr & $\begin{array}{l}\text { deux rochers, } \\
\text { deux stèles }\end{array}$ & $\begin{array}{l}\text { rochers } \\
\text { errants; la } \\
\text { mer passe } \\
\text { sous l'île }\end{array}$ & olivier sacré & serpent tué & $\begin{array}{l}\text { une source } \\
\text { jaillit }\end{array}$ \\
\hline Lérins & $\begin{array}{l}\text { deux petites } \\
\text { îles }\end{array}$ & $\begin{array}{l}\text { au moins une } \\
\text { île errante }\end{array}$ & $\begin{array}{l}\text { serpents } \\
\text { chassés }\end{array}$ & $\begin{array}{l}\text { des sources } \\
\text { jaillissent }\end{array}$ \\
\hline Gadès & $\begin{array}{l}\text { deux îles } \\
\text { deux stèles }\end{array}$ & $\begin{array}{l}\text { la mer passe } \\
\text { sur et sous } \\
\text { l'île }\end{array}$ & olivier sacré & $\begin{array}{l}\text { monstre } \\
\text { pisciforme tué }\end{array}$ & $\begin{array}{l}\text { une source } \\
\text { dans le } \\
\text { sanctuaire }\end{array}$ \\
\hline Lixus & $\begin{array}{l}\text { la mer } \\
\text { pénètre sous } \\
\text { la terre }\end{array}$ & $\begin{array}{l}\text { arbres aux } \\
\text { pommes d'or }\end{array}$ & serpent tué & \\
\hline
\end{tabular}

Bien des questions restent en suspens, et les incertitudes l'emportent encore très largement sur les faits avérés. On me reprochera

${ }^{141}$ Strabon, XVII 3, 3.

${ }_{142}$ Philostrate, Vie d'Apollonius, V 5.

${ }_{143}$ Pausanias, X 4, 6 (récit emprunté à un certain Cléon de Magnésie; voir les commentaires de C. Bonnet, op. cit., p. 222).

144 Pline, Hist. nat., V 3.

${ }_{145}$ Deux îles principales, Érythie et Kotinoussa, auxquelles s'ajoutaient plusieurs îlots. 
sans doute d'avoir échafaudé hypothèses sur hypothèses. Est-il pertinent de mettre sur le même plan des récits structurés (à Tyr et à Lérins) et des collections de données isolées qui peuvent n'avoir aucun lien entre elles (à Lixus et à Gadès)? On peut bien sûr en douter. Et sommes-nous en mesure d'affirmer que le cadre de référence est partout le même, ou qu'il dérive d'un archétype commun? Il faut convenir que non. L'analogie des structures mythiques rend leur parenté vraisemblable, mais elle ne la prouve pas.

Un fait du moins me paraît assuré : c'est l'existence d'une nébuleuse mythique, répandue dans toute la Méditerranée, qui gravitait autour du thème de l'île erratique, ferment de chaos, fille insaisissable des flots, qu'il incomba à un dieu ou à un héros de fixer pour qu'elle fût ainsi rendue à l'ordre du monde. Par cet acte fondateur - au sens le plus concret du mot - , le dieu (Zeus ou Poseidon à Délos, Héraclès-Melqart à Tyr) offre aux hommes l'assise stable qui permettra à leurs établissements de prospérer. Il s'agit donc d'un acte d'appropriation civilisatrice, comme l'atteste la symbolique transparente des deux gestes qui l'accompagnent : tuer le serpent, faire sourdre l'eau douce. Il s'agit d'arracher l'île à ses deux ressorts inhumains, les puissances souterraines et la «mer inféconde». Qui mieux qu'Héraclès, le civilisateur de l'Occident, pouvait incarner cette mission? Lui qui «entra dans l'Olympe après avoir exploré toutes les régions de la terre, tous les abîmes de la mer, aux vagues blanchissantes, aux bords escarpés, et pacifié la route des navigateurs ${ }^{146}$.

Revenons, pour conclure, sur les points communs aux trois îles Planésies que je signalais à mi-chemin. Difficiles à repérer, se détachant mal sur l'horizon en raison de leur faible élévation, elles étaient de mauvais repères; aperçues lors d'un premier passage, on pouvait les manquer et les imaginer parties au voyage suivant. Prolongées par des hauts-fonds, leurs limites mêmes étaient imprécises. Bordées d'écueils, elles étaient dangereuses. Tout concourait donc à les rapporter, dans l'esprit d'un navigateur, à la part la plus hostile et la plus déroutante de l'élément marin; tout concourait à ce qu'on reconnût en elles les îles erratiques de la légende. Situées sur des routes fréquentées dès l'époque archaïque par les bateaux grecs, il était également naturel qu'on associât à leur enracinement la figure d'un Héraclès civilisateur. Quelle fut, dans ce processus d'élaboration et de diffusion occidentale du mythe, la part des Phocéens, puis des Marseillais? Il est impossible de le dire, tant

${ }^{146}$ Pindare, Isthmiques, IV 55-58 (trad. Puech). 
nous connaissons mal les cultes et les mythes de la diaspora phocéenne. Certes, j'ai souligné le lien troublant qui semble rattacher chaque île Planésie à une colonie phocéenne ou, dans le cas ibérique, à un établissement commercial fréquenté par les Grecs. Mais on ne peut pour le moment que constater cette proximité, faute d'indices épigraphiques ou littéraires nous assurant que les Phocéens étaient - comme les Phéniciens, leurs voisins dans l'ouest de la Méditerranée - familiers du mythe de l'île errante.

Pierre MORET, CNRS, UMR 5608, Toulouse - Le Mirail. 